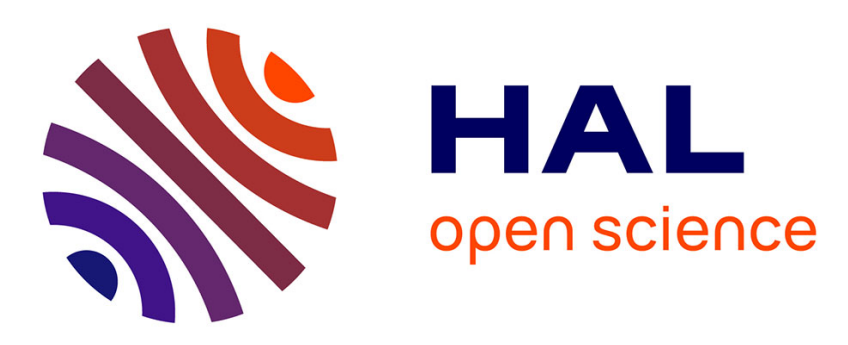

\title{
The Quasi Quantum Treatment of rotationally inelastic scattering from a hard shell potential; Its derivation and practical use
}

\author{
Albert Ballast, Arjan Gijsbertsen, Harold Linnartz, Steven Stolte
}

\section{- To cite this version:}

Albert Ballast, Arjan Gijsbertsen, Harold Linnartz, Steven Stolte. The Quasi Quantum Treatment of rotationally inelastic scattering from a hard shell potential; Its derivation and practical use. Molecular Physics, 2008, 106 (02-04), pp.315-331. 10.1080/00268970701803109 . hal-00513167

\author{
HAL Id: hal-00513167 \\ https://hal.science/hal-00513167
}

Submitted on 1 Sep 2010

HAL is a multi-disciplinary open access archive for the deposit and dissemination of scientific research documents, whether they are published or not. The documents may come from teaching and research institutions in France or abroad, or from public or private research centers.
L'archive ouverte pluridisciplinaire $\mathbf{H A L}$, est destinée au dépôt et à la diffusion de documents scientifiques de niveau recherche, publiés ou non, émanant des établissements d'enseignement et de recherche français ou étrangers, des laboratoires publics ou privés. 


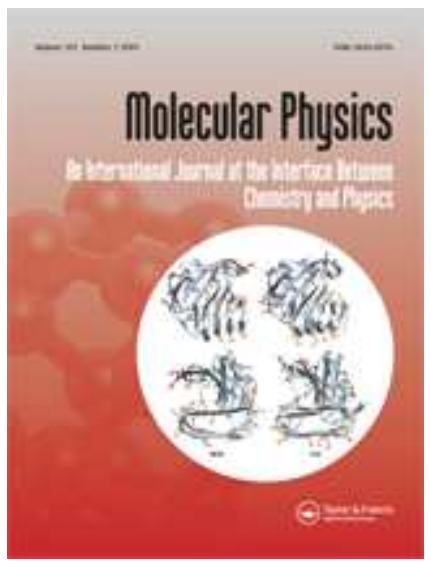

\section{The Quasi Quantum Treatment of rotationally inelastic scattering from a hard shell potential; Its derivation and practical use}

\begin{tabular}{|c|c|}
\hline Journal: & Molecular Physics \\
\hline Manuscript ID: & TMPH-2007-0288.R1 \\
\hline Manuscript Type: & Invited Article \\
\hline $\begin{array}{r}\text { Date Submitted by the } \\
\text { Author: }\end{array}$ & 09-Nov-2007 \\
\hline Complete List of Authors: & $\begin{array}{l}\text { Ballast, Albert; Vrije Universiteit Amsterdam, Physical Chemistry } \\
\text { and Laser Centre } \\
\text { Gijsbertsen, Arjan; FOM Institute for Atomic and Molecular Physics } \\
\text { (AMOLF) } \\
\text { Linnartz, Harold; Leiden Observatory Ẫ University of Leiden, } \\
\text { Sackler laboratory for Astrophysics } \\
\text { Stolte, Steven; Vrije Universiteit Amsterdam, Physical Chemistry } \\
\text { and Laser Centre }\end{array}$ \\
\hline Keywords: & $\begin{array}{l}\text { molecular collisions, differential cross-sections, hardshell potential, } \\
\text { rotational excitation, } \mathrm{Ne}-\mathrm{CO}, \mathrm{He}-\mathrm{NO}\end{array}$ \\
\hline \multicolumn{2}{|c|}{$\begin{array}{l}\text { Note: The following files were submitted by the author for peer review, but cannot be converted } \\
\text { to PDF. You must view these files (e.g. movies) online. }\end{array}$} \\
\hline $\begin{array}{l}\text { Ballast_etal-QQT---12nov200 } \\
\text { arjan-ab.bib }\end{array}$ & 7.tex \\
\hline
\end{tabular}




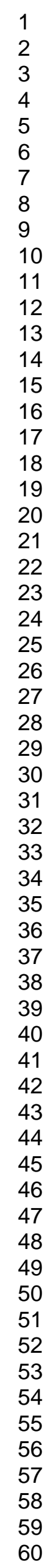

\section{$\$$ Manuscript Central}

URL: http://mc.manuscriptcentral.com/tandf/tmph 


\title{
The Quasi Quantum Treatment of rotationally inelastic scattering from a hard shell potential Its derivation and practical use
}

\author{
Albert Ballast \\ Laser Centre and Department of Physical Chemistry, Vrije Universiteit Amsterdam, \\ De Boelelaan 1083, 1081 HV Amsterdam, The Netherlands \\ Arjan Gijsbertsen \\ FOM Institute for Atomic and Molecular Physics (AMOLF), \\ Kruislaan 407, 1098 SJ Amsterdam, The Netherlands \\ Harold Linnartz \\ Sackler laboratory for Astrophysics, Leiden Observatory, \\ P.O. Box 9513, 2300 RA Leiden, The Netherlands \\ Steven Stolte ${ }^{\dagger}$ \\ Laser Centre and Department of Physical Chemistry, Vrije Universiteit Amsterdam, \\ De Boelelaan 1083, 1081 HV Amsterdam, The Netherlands
}

(Dated: November 12, 2007)

$\dagger^{\dagger}$ Author to whom correspondence should be addressed, fax +31205987643 , e-mail stolte@few.vu.nl 


\begin{abstract}
The QQT is a quasi-quantum mechanical treatment of the collision between molecules. Instead of a partial wave expansion approach, it uses a kind of Feynman path integral method that exploits the path length differences originating from the different orientations of an anisotropic molecule. As a result the QQT provides valuable physical insight while requiring very little computational effort. The current paper gives a systematic derivation of the QQT and explains its underlying principles. The expression for the scattering amplitude is shown to be self-consistent, without any normalisation factors, when the rotational energy level spacing is negligible. The constant curvature approximation that is presented makes the QQT conceptually even more simple, and its effect on the calculated differential cross-sections (DCS's) turns out to be small. As examples we present QQT calculations of the DCS's for $\mathrm{Ne}-\mathrm{CO}\left({ }^{1} \Sigma\right)$ and for $\mathrm{He}-\mathrm{NO}\left({ }^{2} \Pi\right)$, at collision energies of respectively $511 \mathrm{~cm}^{-1}$ and $514 \mathrm{~cm}^{-1}$. The anisotropy of the hardshell potential energy surface for $\mathrm{Ne}-\mathrm{CO}$ in terms of the incoming de Broglie wavelength is about twice as large as for He-NO. This leads to state-to-state DCS's that have up to three maxima of comparable amplitude, instead of only one large maximum as is found for He-NO. The QQT results for these two applications are compared to results from close coupling calculations.
\end{abstract}

Keywords: molecular collisions; differential cross-sections; hardshell potential; rotational excitation; Ne-CO; He-NO; 


\section{INTRODUCTION}

In a previous paper ${ }^{1}$ the Quasi Quantum mechanical Treatment (QQT) approach to inelastic collisions was introduced. The present work gives a systematic derivation that will clarify the physical basis and the underlying assumptions of the QQT. A new constant curvature approximation is introduced and the normalisation factor in the differential crosssection is now shown to approach unity in the limit of vanishing rotational energy level spacing. The two selected applications are complementary: $\mathrm{Ne}-\mathrm{CO}\left({ }^{1} \Sigma\right)$ is conceptually more simple than He- $\mathrm{NO}\left({ }^{2} \Pi\right)$, but its differential cross-sections are more complicated. Both $\mathrm{Ne}-\mathrm{CO}^{2-6}$ and $\mathrm{He}-\mathrm{NO}^{7-20}$ have been studied extensively in the literature, experimentally as well as theoretically.

The development of QQT benefited greatly from the pioneering work of Evans and Hoffman. In a seminal study Hoffman ${ }^{21}$ developed a classical kinetic theory for a mixture of dilute gases of rigid convex molecules in which molecular orientation angles, instead of impact parameters, were used to evaluate the collision integrals. Evans and co-workers ${ }^{22,23}$ succeeded to apply Hoffman's angular approach to the calculation of the classical bimolecular rate constant, the energy dependent reaction cross-section, and the steric dependence of the differential cross-sections for general diatom-diatom collisions. Another ingredient was presented by Khare et al., ${ }^{24,25}$ who showed explicitly that there is a propensity for preserving the angular momentum component along the kinematic apse.

The QQT was first introduced by Gijsbertsen et al. ${ }^{1}$ in the context of rotational inelastic scattering of rare gases from oriented NO molecules. They were able to explain the remarkable fact that $\mathrm{N}$-end (O-end) collisions are favoured for odd (even) changes in the rotational quantum number ${ }^{16,19,26,27}$. In addition, the existence of so-called parity pairs in the differential cross-sections was predicted by the QQT calculations and confirmed both experimentally and by exact quantum mechanical calculations. Very recently, Taatjes et al. ${ }^{19}$ used the QQT to obtain a hard shell potential energy surface for $\mathrm{NO}-\mathrm{D}_{2}$ by a fitting procedure using experimental data for the steric asymmetry of the integral cross-section. The QQT calculations of the differential cross-sections using this fitted potential show reasonable agreement with the measured differential cross-sections. 


\section{THEORY OF QQT}

We consider a closed shell atom that collides with a diatomic molecule. The resulting scattering may be inelastic, since the rotational state of the diatomic can change. The interaction potential between the atom $\mathrm{A}$ and the molecule $\mathrm{BC}$ is described by an anisotropic potential energy surface. This PES only depends on the displacement vector $\boldsymbol{R}=\boldsymbol{R}_{A}-\boldsymbol{R}_{B C}$ of the centre of A with respect to the centre of mass of BC and on the Jacobi angle $\gamma_{R}$ between the molecular axis $\hat{\boldsymbol{r}}$ and the displacement vector $\boldsymbol{R}$, with $\cos \gamma_{R}=\hat{\boldsymbol{r}} \cdot \hat{\boldsymbol{R}}$. The Hamiltonian of this rotationally inelastic scattering problem in the centre of mass system can be written $\operatorname{as}^{28}$

$$
H=-\frac{\hbar^{2}}{2 \mu} \nabla_{R}^{2}+V\left(R, \gamma_{R}\right)+H_{\text {rot }}
$$

with $\mu$ the reduced mass $m_{A} m_{B C} /\left(m_{A}+m_{B C}\right)$. The potential energy surface is approximated by a hardshell potential ${ }^{29}$ : inside the equipotential contour $R_{\mathrm{S}}\left(\gamma_{R}\right)$, defined by $V\left(R_{\mathrm{S}}\left(\gamma_{R}\right), \gamma_{R}\right)=E_{\text {coll }}$, the hardshell potential is infinite and outside this contour it is zero. 
The interaction is then limited to the short moment of contact at the point of closest approach. For the details see appendix A.

The standard approach ${ }^{30,31}$ to solving this scattering problem is to use a partial wave analysis in which the wave function is expanded in the eigen functions of the orbital angular momentum operator $L$, with eigen values $l$ that in the classical limit can be identified as the impact parameters $b$. The resulting set of coupled differential equations is large and requires a lot of computational effort to solve. In the QQT however, we do not need the $l$ quantum number. Instead, we assign a phase factor to each (classical) scattering trajectory according to its relative path length and the de Broglie wavelength. For an anisotropic potential this path length depends on the orientation of the molecular axis at the time of the collision. For a given scattering angle this creates a "phase factor function". Multiplying the initial rotational wave function with this phase factor function gives a new function that has, in general, a non-vanishing overlap with several possible final rotational states. This overlap integral is taken to be the scattering amplitude and its absolute square gives the different branching fractions. These are then normalised to the geometrical cross-section of the hardshell. In essence, we are using a Feynman path integral approach, since we integrate over the probability amplitudes of all the indistinguishable scattering possibilities. However, we only consider the trajectories that hit the hardshell. Diffraction effects or, in other words, grazing trajectories that deflect because of the shadow left by the hardshell, are not taken into account.

[Figure 1 about here.]

All the vectors and angles relevant for the hardshell collision problem are shown in figure 1. The primary reference direction is the direction of the incoming wave vector $\boldsymbol{k}$. The outgoing wave vector is $\boldsymbol{k}^{\prime}$ and it makes an angle $\theta$ with the incoming wave vector. Its azimuthal angle is $\phi$. The momentum transferred in the collision is equal to $\Delta \boldsymbol{p}=\boldsymbol{p}^{\prime}-\boldsymbol{p}=\hbar \boldsymbol{k}^{\prime}-\hbar \boldsymbol{k}$. The unit vector in the direction of this $\Delta \boldsymbol{p}$ is called the (kinematic) apse and is given by ${ }^{24,25}$

$$
\hat{\boldsymbol{a}}=\frac{\boldsymbol{k}^{\prime}-\boldsymbol{k}}{\left|\boldsymbol{k}^{\prime}-\boldsymbol{k}\right|}
$$

We define

$$
a=k^{\prime}-k
$$


The component of the outgoing wave vector perpendicular to the surface is pointing away from the surface, $\hat{\boldsymbol{k}}_{\perp}^{\prime}=-\hat{\boldsymbol{k}}_{\perp}$, and its magnitude is determined by the amount of translational energy lost to rotational excitation:

$$
\begin{gathered}
k_{\perp}=|k \cos \beta| \\
k_{\perp}^{\prime}=k_{\perp} \sqrt{1-\Delta E_{\mathrm{rot}} /\left(\frac{\hbar^{2} k_{\perp}^{2}}{2 \mu}\right)}
\end{gathered}
$$

with $\Delta E_{\text {rot }}=E_{\text {rot }}\left(j^{\prime}\right)-E_{\text {rot }}(j)$, see appendix B. Transitions with $\Delta E_{\text {rot }}>\frac{\hbar^{2} k_{\perp}^{2}}{2 \mu}$ are not allowed. Note that changing $\Delta E_{\text {rot }}$ will alter the angle $\theta$ that $\boldsymbol{k}^{\prime}$ makes with $\boldsymbol{k}$, but that it will not alter the angle $\beta$ that $\hat{\boldsymbol{a}}$ makes with $\boldsymbol{k}$. Given $\Delta E_{\text {rot }}$, there is a unique relation between the direction of the apse and the direction of the outgoing wave vector:

$$
\theta=\beta-\arctan \frac{k_{\|}^{\prime}}{k_{\perp}^{\prime}}
$$

It is instructive to consider the limits $k_{\perp}^{\prime}=0$ (completely inelastic) and $k_{\perp}^{\prime}=k_{\perp}$ (elastic): $\theta$ will be respectively $\beta-\frac{1}{2} \pi$ and $2 \beta-\pi$; note the factor of two between these two angles. Later in this section we will show that the projection of the angular momentum along the apse is conserved. That is one of the reasons why we let the apse play such a central role in the following formulation of the scattering problem.

[Figure 2 about here.]

To calculate, later in this section, the phase shift and the geometrical properties at the point of contact, we need to know the direction of $\boldsymbol{R}$ when the direction of $\hat{\boldsymbol{r}}$ is given. The molecular axis $\hat{\boldsymbol{r}}$ makes an angle $\gamma_{a}$ with the apse $\hat{\boldsymbol{a}}$ and its azimuthal angle with respect to 
that apse is $\phi_{a}$. The vector $\boldsymbol{R}$ points from the centre of mass of the hardshell to the point of contact and makes an angle $\gamma_{R}$ with the molecular axis $\hat{\boldsymbol{r}}$ while its azimuthal angle with respect to that axis is $\phi_{R}$. Since the hardshell is cylindrical symmetric the vectors $\hat{\boldsymbol{r}}, \boldsymbol{R}$, and $\boldsymbol{a}$ are always in one plane. This makes it possible to specify their relative orientation using only the angles $\gamma_{a}$ and $\gamma_{R}$. When $\phi_{a}=0$ or $\pi$ their plane coincides with the plane containing $\boldsymbol{k}, \boldsymbol{k}^{\prime}$, and $\boldsymbol{a}$; see also figure 2. For a convex hardshell there is a unique relation between the angle $\gamma_{a}$ and the angle $\gamma_{R}$. Specifying the direction of the molecular axis with respect to the apse therefore implies specifying the point of contact on the hardshell and this point of contact is independent of the azimuthal angles $\phi_{a}$ and $\phi_{R}$ (to be precise: changing $\phi_{R}$ will give a different, but equivalent point of contact). To find an explicit expression for the relation between the angle $\gamma_{a}$ and the angle $\gamma_{R}$, it is convenient to rewrite the hardshell in terms of cylinder coordinates $\left(z_{\mathrm{cyl}}, r_{\mathrm{cyl}}\right)$, with the $z_{\mathrm{cyl}}$-axis coinciding with the molecular axis $\hat{\boldsymbol{r}}$ :

$$
\begin{gathered}
z_{\mathrm{cyl}}=R_{\mathrm{S}}\left(\gamma_{R}\right) \cos \gamma_{R} \\
r_{\mathrm{cyl}}=R_{\mathrm{S}}\left(\gamma_{R}\right) \sin \gamma_{R}
\end{gathered}
$$

For a hardshell, the apse is pointing in the same direction as the surface normal, so the angle $\gamma_{a}$ follows from the angle $\gamma_{R}$ via

$$
\gamma_{a}=\arctan \left(\frac{d r_{\mathrm{cyl}}}{d z_{\mathrm{cyl}}}\right)+\frac{\pi}{2}
$$

In the calculations we need $\gamma_{R}$ as function of $\gamma_{a}$ and equation (11) is therefore inverted numerically to give $\gamma_{R}\left(\gamma_{a}\right)$. Now we are ready to introduce the differential cross-section in the apse frame, but we start first with the standard differential cross-section.

The outcome of a scattering experiment is described in terms of the state-to-state differential cross-section $d \sigma_{i \rightarrow f} / d \omega(\theta, \phi)$. It gives the amount of target area $d \sigma_{i \rightarrow f}$ that scatters incoming rotational states $i$ as outgoing rotational states $f$ into the cone $d \omega=\sin \theta d \theta d \phi$ pointing in the direction of $(\theta, \phi)$. The differential cross-section is equal to the modulus squared of what is called the dimensionless ${ }^{44}$ scattering amplitude:

$$
\frac{d \sigma_{i \rightarrow f}}{d \omega}(\theta, \phi)=\frac{1}{k^{2}}\left|f_{i \rightarrow f}(\theta, \phi)\right|^{2}
$$


Since specifying the direction of the apse $\hat{\boldsymbol{a}}$ specifies the plane of contact, but not the amount of energy lost to rotational excitation, it is very convenient to formulate the differential crosssection with respect to the direction of the apse $\hat{\boldsymbol{a}}$ instead of with respect to the direction of the outgoing wave vector $\boldsymbol{k}^{\prime}$. It is not only convenient however, it also makes it possible to normalise the branching fractions to the geometrical cross-section of the hardshell. We will therefore use the spherical angles $(\beta, \alpha)$ instead of the spherical angles $(\theta, \phi)$ and the element of solid angle $d \omega_{a}$ instead of the element of solid angle $d \omega$. The scattering amplitude in this apse frame is denoted by $g_{i \rightarrow f}(\beta, \alpha)$ :

$$
\frac{d \sigma_{i \rightarrow f}}{d \omega_{a}}(\beta, \alpha)=\frac{1}{k^{2}}\left|g_{i \rightarrow f}(\beta, \alpha)\right|^{2}
$$

If the probability distribution for the direction of the molecular axis $\hat{\boldsymbol{r}}$ with respect to the incoming wave vector $\boldsymbol{k}$ is cylindrical symmetric, then the dependence on $\phi$ and $\alpha$ can be ignored and we will do so. The standard differential cross-section can then be written in terms of the scattering amplitude in the apse frame as follows

$$
\left.\frac{d \sigma_{i \rightarrow f}}{d \omega}=\frac{d \sigma_{i \rightarrow f}}{d \omega_{a}} \frac{d \omega_{a}}{d \omega}=\frac{1}{k^{2}} \mid g_{i \rightarrow f}(\beta)\right)\left.\right|^{2} \frac{\sin \beta}{\sin \theta} \frac{d \beta}{d \theta}
$$

where the expression $\frac{d \omega_{a}}{d \omega}$ is the Jacobian for this coordinate transformation.

We will first give the scattering amplitude for a single, fixed orientation of the molecular axis. Later we then use this expression in an integral over all possible orientations. For a transition from an initial rotational state $i$ to a final rotational state $f$ the scattering amplitude $g_{i \rightarrow f}$ in the apse frame for the (hypothetical) case that the molecule has a certain fixed direction of its molecular axis is written $g_{i \rightarrow f}\left(\gamma_{a} ; \beta\right)$ and we refer to it as the "molecule fixed scattering amplitude". It has a phase part $e^{i \eta_{i \rightarrow f}\left(\gamma_{a} ; \beta\right)}$ that will give the interference between the contributions from the different directions of the molecular axis. And it has a magnitude $g_{\text {geom }}\left(\gamma_{a} ; \beta\right)=\left|g_{i \rightarrow f}\left(\gamma_{a} ; \beta\right)\right|$ that, when squared, should be equal to the "classical" or "geometrical" differential cross-section for a fixed molecule, see also appendix C or equation (19):

$$
g_{\text {geom }}\left(\gamma_{a} ; \beta\right)=k \sqrt{\frac{d \sigma^{\text {geom }}}{d \omega_{a}}\left(\gamma_{a} ; \beta\right)}
$$

In the apse frame $g_{\text {geom }}\left(\gamma_{a} ; \beta\right)$ is independent of the initial and of the final rotational state. Next we turn to the phase part of the scattering amplitude. Assigning a phase difference of 
zero to the virtual trajectory through the origin we can write

$$
\eta_{i \rightarrow f}\left(\gamma_{a} ; \beta\right)=-\boldsymbol{a} \cdot \boldsymbol{R}=-\left(k_{\perp}+k_{\perp}^{\prime}\right) R_{\mathrm{S}}\left(\gamma_{R}\right) \cos \left(\gamma_{a}-\gamma_{R}\right)
$$

where $\boldsymbol{R}$ is the distance from the origin to the point of contact, see also figure 3 . This phase shift is independent of $\phi_{a}$.

[Figure 3 about here.]

Now we can add up all the separate contributions from all the possible orientations of the molecular axis. The rotational wave function gives the probability amplitude to find the molecule with its molecular axis pointing in a certain direction. To get the scattering amplitude $g_{i \rightarrow f}(\beta)$ we therefore have to integrate the molecule fixed scattering amplitude $g_{i \rightarrow f}\left(\gamma_{a} ; \beta\right)$ over all the possible directions of the molecular axis (angles $\gamma_{a}$ and $\phi_{a}$ ) while taking into account the probability amplitudes for those directions in both the initial and in the final rotational wave function:

$$
g_{i \rightarrow f}(\beta)=C(\beta) A_{i \rightarrow f}(\beta) \int_{0}^{2 \pi} \int_{0}^{\pi} g_{\text {geom }}\left(\gamma_{a} ; \beta\right) e^{i \eta_{i \rightarrow f}\left(\gamma_{a} ; \beta\right)} \Psi_{f}^{*}\left(\gamma_{a}, \phi_{a}\right) \Psi_{i}\left(\gamma_{a}, \phi_{a}\right) \sin \gamma_{a} d \phi_{a} d \gamma_{a}
$$

Here $C(\beta)$ is a normalisation constant that will be discussed later on and $A_{i \rightarrow f}(\beta)$ is equal to one for energetically allowed transitions and equal to zero for energetically not allowed transitions (see also equation (7)):

$$
A_{i \rightarrow f}(\beta)= \begin{cases}0 & \frac{\hbar^{2} k_{\perp}^{2}}{2 \mu}<\Delta E_{\mathrm{rot}} \\ 1 & \frac{\hbar^{2} k_{\perp}^{2}}{2 \mu} \geqslant \Delta E_{\mathrm{rot}}\end{cases}
$$

If we use the apse as the quantization axis, the product of the wave functions $\Psi_{f}^{*}\left(\gamma_{a}, \phi_{a}\right) \Psi_{i}\left(\gamma_{a}, \phi_{a}\right)$ depends on $\phi_{a}$ as $e^{i\left(m_{f}-m_{i}\right) \phi_{a}}$. The other parts of the integrand, $g_{\text {geom }}\left(\gamma_{a} ; \beta\right)$ and $e^{i \eta_{i \rightarrow f}\left(\gamma_{a} ; \beta\right)}$, do not depend on $\phi_{a}$. Since the difference $\left(m_{f}-m_{i}\right)$ of the projection quantum numbers is always an integer, the integral over $\phi_{a}$ in equation (17) will vanish unless $m_{f}=m_{i}$. The integral over $\phi_{a}$ can therefore be replaced by a factor of $2 \pi \delta_{m_{f}, m_{i}}$. This shows that for a hardshell potential the projection of the angular momentum along the apse is conserved in the collision. 
In appendix $\mathrm{C}$ the molecule fixed geometrical differential cross-section is derived. Here we will use that result to calculate the geometrical differential cross-section of the molecule using its rotational wave function. From this result then follow some attractive approximations of the molecule fixed geometrical differential cross-section. In appendix $\mathrm{C}$ it is shown that the angular extent of a cone $d \omega_{a}$ is determined by the amount of curvature at the point of contact. The resulting molecule fixed geometrical cross-section is

$$
\frac{d \sigma^{\text {geom }}}{d \omega_{a}}\left(\gamma_{a} ; \beta\right)=|\cos \beta| \rho_{1}\left(\gamma_{a}\right) \rho_{2}\left(\gamma_{a}\right)
$$

with $\rho_{1}\left(\gamma_{a}\right), \rho_{2}\left(\gamma_{a}\right)$ the local radii of curvature of the hardshell, for their calculation see again appendix C. The geometrical differential cross section for a hardshell having a rotational wave function $\Psi_{i}\left(\gamma_{a}, \phi_{a}\right)$ is then obtained by integrating equation (19) over all possible orientations of the molecular axis, taking into account the probability for each orientation. These probabilities are given by the absolute square of that incoming rotational wave function, $\left|\Psi_{i}\left(\gamma_{a}, \phi_{a}\right)\right|^{2}$. The resulting expression is

$$
\begin{aligned}
\frac{d \sigma^{\text {geom }}}{d \omega_{a}}(\beta) & =|\cos \beta| \int_{0}^{2 \pi} \int_{0}^{\pi}\left|\Psi_{i}\left(\gamma_{a}, \phi_{a}\right)\right|^{2} \rho_{1}\left(\gamma_{a}\right) \rho_{2}\left(\gamma_{a}\right) \sin \gamma_{a} d \phi_{a} d \gamma_{a} \\
& =|\cos \beta| R_{\mathrm{eff}}^{2}
\end{aligned}
$$

The last step defines the effective radius of curvature $R_{\text {eff }}$; it is also the radius of the sphere that has the same total geometrical cross-section as the hardshell with its rotational wave function. If the variation in the local curvature (as function of $\gamma_{a}$ ) is small we can use the following constant curvature approximation for the molecule fixed geometrical differential cross-section:

$$
\frac{d \sigma^{\text {geom }}}{d \omega_{a}}\left(\gamma_{a} ; \beta\right) \approx|\cos \beta| R_{\mathrm{eff}}^{2}
$$

With such a constant curvature assumption the molecule fixed scattering amplitude $g_{\text {geom }}\left(\gamma_{a} ; \beta\right)$ is equal to $g_{\text {geom }}(\beta)$ and can be taken out of the integral in equation (17). It can then be included in the normalisation constant $C(\beta)$. To calculate $R_{\text {eff }}$ we still need the local curvatures. If we do not want to bother with those we can also use

$$
\frac{d \sigma^{\text {geom }}}{d \omega_{a}}\left(\gamma_{a} ; \beta\right) \approx|\cos \beta| R_{0}^{2}
$$


where $R_{0}$ is the average radius of the hardshell, the zeroth order term in the expansion given in equation (A1). When using $R_{0}$ the total geometrical cross-section differs from the value it has when using the full curvature. ${ }^{45}$

As the last step we introduce the normalisation of the branching fractions to the geometrical differential cross-section. The factor $C(\beta)$ is determined implicitly by the requirement that the state-to-state differential cross-section summed over all outgoing channels should be equal to the geometrical differential cross-section (see equation (20)):

$$
\sum_{\text {all } f} \frac{d \sigma_{i \rightarrow f}}{d \omega_{a}}(\beta)=\frac{d \sigma^{\text {geom }}}{d \omega_{a}}(\beta)
$$

Using equations (13) and (17)

$$
C^{2}(\beta)=\frac{d \sigma^{\text {geom }}}{d \omega_{a}}(\beta) \cdot \frac{k^{2}}{\sum_{\text {all } f} A_{i \rightarrow f}(\beta)\left|\int_{0}^{2 \pi} \int_{0}^{\pi} g_{\text {geom }}\left(\gamma_{a} ; \beta\right) e^{i \eta_{i \rightarrow f}\left(\gamma_{a} ; \beta\right)} \Psi_{f}^{*}\left(\gamma_{a}, \phi_{a}\right) \Psi_{i}\left(\gamma_{a}, \phi_{a}\right) \sin \gamma_{a} d \phi_{a} d \gamma_{a}\right|^{2}}
$$

Or, using equation (21)

$$
C^{2}(\beta)=\frac{1}{\sum_{\text {all } f} A_{i \rightarrow f}(\beta)\left|\int_{0}^{2 \pi} \int_{0}^{\pi} \frac{g_{\mathrm{geom}}\left(\gamma_{a} ; \beta\right)}{k R_{\mathrm{eff}} \sqrt{|\cos \beta|}} e^{i \eta_{i \rightarrow f}\left(\gamma_{a} ; \beta\right)} \Psi_{f}^{*}\left(\gamma_{a}, \phi_{a}\right) \Psi_{i}\left(\gamma_{a}, \phi_{a}\right) \sin \gamma_{a} d \phi_{a} d \gamma_{a}\right|^{2}}
$$

The denominator in equation (26) is equal to one in the limit of vanishing rotational energy level spacing, see appendix E. Using equations (13), (17), and (25), the final expression for the differential cross-section in the apse frame becomes

$$
\frac{d \sigma_{i \rightarrow f}}{d \omega_{a}}(\beta)=\frac{d \sigma^{\text {geom }}}{d \omega_{a}}(\beta) \cdot \frac{A_{i \rightarrow f}(\beta)\left|\int_{0}^{2 \pi} \int_{0}^{\pi} g_{\text {geom }}\left(\gamma_{a} ; \beta\right) e^{i \eta_{i \rightarrow f}\left(\gamma_{a} ; \beta\right)} \Psi_{f}^{*}\left(\gamma_{a}, \phi_{a}\right) \Psi_{i}\left(\gamma_{a}, \phi_{a}\right) \sin \gamma_{a} d \phi_{a} d \gamma_{a}\right|^{2}}{\sum_{\text {all } f} A_{i \rightarrow f}(\beta)\left|\int_{0}^{2 \pi} \int_{0}^{\pi} g_{\text {geom }}\left(\gamma_{a} ; \beta\right) e^{i \eta_{i \rightarrow f}\left(\gamma_{a} ; \beta\right)} \Psi_{f}^{*}\left(\gamma_{a}, \phi_{a}\right) \Psi_{i}\left(\gamma_{a}, \phi_{a}\right) \sin \gamma_{a} d \phi_{a} d \gamma_{a}\right|^{2}}
$$

In the constant curvature approximation $g_{\text {geom }}\left(\gamma_{a} ; \beta\right)$ does not depend on $\gamma_{a}$ and its occurrences in the numerator and the denominator cancel. The expression for the standard differential cross-section follows from equation (14). Note that the formulation of the QQT differential cross-section in equation (27) is chosen such that the structure "geometrical cross-section times branching fraction" can be recognized more easily. 


\section{APPLICATION TO ${ }^{1} \Sigma$ MOLECULES: NE COLLIDING WITH CO}

$\mathrm{CO}$ is a ${ }^{1} \Sigma$ molecule. Its rotational wave function is that for a linear rotor. The eigenfunctions are given by the spherical harmonics $Y_{j m}$. We consider transitions from the ground state of $\mathrm{CO}$ to rotationally excited states induced by the collisions with Ne. In the ground state the rotational wave function is given by

$$
\Psi_{00}=Y_{00}=\sqrt{\frac{1}{4 \pi}}
$$

We have shown that for a hardshell the component of the angular momentum along the apse is conserved during the collision. This means that the excited state is represented by

$$
\Psi_{j^{\prime} 0}=Y_{j^{\prime} 0}=\sqrt{\frac{2 j^{\prime}+1}{4 \pi}} P_{j^{\prime}}\left(\cos \gamma_{a}\right)
$$

where $P_{j^{\prime}}$ is a Legendre polynomial of order $j^{\prime}$. The product of the two rotational wave functions is now

$$
\Psi_{j^{\prime} 0}^{*} \Psi_{00}=\frac{\sqrt{2 j^{\prime}+1}}{4 \pi} P_{j^{\prime}}\left(\cos \gamma_{a}\right)
$$

In the apse frame the scattering amplitude then becomes (after performing the trivial integration over $\left.\phi_{a}\right)$

$$
g_{j^{\prime}}(\beta)=C(\beta) \frac{\sqrt{2 j^{\prime}+1}}{2} \int_{0}^{\pi} g_{\text {geom }}\left(\gamma_{a} ; \beta\right) e^{i \eta_{j^{\prime}}\left(\gamma_{a} ; \beta\right)} P_{j^{\prime}}\left(\cos \gamma_{a}\right) \sin \gamma_{a} d \gamma_{a}
$$

giving the differential cross-section (now assuming constant curvature)

$$
\frac{d \sigma_{0 \rightarrow j^{\prime}}}{d \omega_{a}}(\beta)=\frac{d \sigma^{\text {geom }}}{d \omega_{a}}(\beta) \cdot \frac{\frac{\left(2 j^{\prime}+1\right)}{4}\left|\int_{0}^{\pi} e^{i \eta_{j^{\prime}}\left(\gamma_{a} ; \beta\right)} P_{j^{\prime}}\left(\cos \gamma_{a}\right) \sin \gamma_{a} d \gamma_{a}\right|^{2}}{\sum_{\text {all } j^{\prime}} \frac{\left(2 j^{\prime}+1\right)}{4}\left|\int_{0}^{\pi} e^{i \eta_{j^{\prime}}\left(\gamma_{a} ; \beta\right)} P_{j^{\prime}}\left(\cos \gamma_{a}\right) \sin \gamma_{a} d \gamma_{a}\right|^{2}}
$$


The phase shift $\eta_{j^{\prime}}\left(\gamma_{a} ; \beta\right)$ depends on the energy levels (and thus on $\left.j^{\prime}\right)$ via the outgoing de Broglie wavelength. The formulas and the constants needed to calculate the energy levels are given in appendix B. In appendix $\mathrm{E}$ it is shown that the sum over all final states in the denominator of equation (32) is equal to one if $\Delta E_{\text {rot }}(\Delta j)=0$ for all $\Delta j$. That means that the expression for the scattering amplitude does not need any normalisation factors in that case.

[Figure 4 about here.]

[Figure 5 about here.]

The hardshell potential energy surface is given in figure 4 and the resulting phase is given in figure 5. In the plot of the potential energy surface the dot in the middle represents the centre of mass of the molecule. The capital letters show which atom is on which side of the potential energy surface. The point where the distance between the hardshell and the centre is the smallest we call the point of nearest contact. Through this point a dotted circle is drawn with its centre at the centre of mass. The radius of this circle is the minimum contact distance. Concentric to this first circle other circles are drawn, each with a radius that is one incoming de Broglie wavelength larger than the previous one. In this way one gets a good idea of the anisotropy of the potential energy surface in terms of this de Broglie wavelength. In the plot of the phase shift the phase shift at the point of nearest contact is set to zero and left and right are the same as for the potential energy surface plot. The bottom one of the phase shift curves is the one corresponding to an elastic collision. The more or less inelastic collisions have absolute phase shifts that are smaller, since the outgoing de Broglie wavelength is smaller. The given phase shift curves are for $\beta=\pi$, which corresponds to strictly backward scattering. For this case the range in phase shifts as function of $\cos \gamma_{a}$ is largest. When $\beta$ goes to $\pi / 2$ the phase shifts go to 0 . The number of periods given by the phase shift curves is twice as large as the number of wavelengths suggested by the dotted circles in the potential energy surface plot. This is because that number of wavelengths holds for both the incoming and the outgoing trajectory and should therefore be counted twice. 
Next we will describe how one can qualitatively reason about the effects of the integral over the phase factor and the Legendre polynomial. Looking at a plot of the Legendre polynomials as function of $\cos \gamma_{a}$ one can see that one can associate a more or less welldefined period or wavelength with each of them. When the period of the phase factor and that of the Legendre polynomial match as function of $\cos \gamma_{a}$, we get a large contribution from the integral and therefore a maximum in the differential cross-section. As said before, the phase shift as function of $\beta$ is largest for backward scattering and goes to zero for glancing collisions. A given Legendre polynomial will therefore give a maximum in the differential cross-section for that angle of $\beta$ for which the range in phase shifts matches the period of this Legendre polynomial. When the range in phase shifts as function of $\cos \gamma_{a}$ is not large enough to match the period of the Legendre polynomial, the maximum will not be reached. If the range in phase shifts is sufficiently large we will also see "higher harmonics" or secondary maxima. The differential cross-section for a rotational transition is zero for glancing collisions, since there is not enough "perpendicular energy" to make the transition possible. Only when the scattering angle has become large enough to make available enough perpendicular energy the differential cross-section starts to be different from zero.

[Figure 6 about here.]

In figure 6 the differential cross-sections from QQT using full curvature are compared to the results of close coupling calculations from McBane, as published in a slightly different form by Lorenz et al. ${ }^{4}$ The computer code he used was MOLSCAT ${ }^{32}$. It is clear that both results are considering the same system, but there are also significant differences, most notably for $\Delta j=8$. The very high differential cross-sections for $\theta<40^{\circ}$ and $\Delta j \leqslant 5$ of the close coupling calculations are mainly due to diffraction effects. Those are not included in the QQT. Another effect that contributes is the extra range of the full softshell with respect to the hardshell ${ }^{33}$. In general the close coupling results reach their maxima at smaller scattering angles relative to the QQT results. This is related to the softness of the full potential energy surface. Also the relative amplitudes of the maxima show some distinct differences between the QQT calculations and the close coupling results. The overall picture of what is happening is however represented quite well by the QQT calculations, especially 
considering the fact that the calculations took only a few seconds on a normal desktop computer.

Very recently, Kłos et al. ${ }^{34}$ noticed that for He-NO collisions at $1186 \mathrm{~cm}^{-1}$ the differential cross-sections show a double peaked structure for the transitions that conserve total parity. In addition they noticed that the position of the dip in the double peaked differential cross-sections matches quite closely that of the peak in the differential cross-sections observed for the next highest $\Delta j$ of opposite parity. The effects were present for both open shell close-coupled quantum mechanical calculations and for closed shell quantum mechanical calculations. For our Ne-CO case the differential cross-sections are also multiply peaked. Here, for $\Delta j=4$ till $\Delta j=9$, the parity conserving transitions show a triple peaked structure and the parity changing transitions a double peaked structure. For the close coupling calculations the effects are not as clear as for the QQT calculations. In the QQT calculations the dips for the parity conserving transitions correspond to the peaks of the next highest $\Delta j$ of opposite parity. This can be attributed to the even/oddness of the Legendre polynomials. In the close coupling calculations the dip-peak correspondence can for example be seen for $\Delta j=4$ and $\Delta j=5$.

[Figure 7 about here.]

In figure 7 the differential cross-sections from QQT using full curvature are compared to the differential cross-sections calculated using the constant curvature approximation. It can be seen that for this application the differences are very small. For the amplitude the differences are less than say $10 \%$ and the differences in the angular dependence are not noticeable. It is clear that for this application the effect of the constant curvature approximation is small compared to the effects of the other basic approximations involved in the QQT calculations, such as neglecting the diffraction and assuming a hardshell potential energy surface.

\section{APPLICATION TO ${ }^{2} \Pi$ MOLECULES: HE COLLIDING WITH NO}

The NO molecule has a ${ }^{2} \Pi$ electronic configuration. We assume that NO can be described as a Hund's case (a) molecule. For small values of $j^{\prime}$ this is a good approximation, see e.g. 
Joswig et al. ${ }^{7}$ The projection of the angular momentum along the molecular axis, $\Omega$, is given by the sum of the electronic spin angular momentum component and the electronic orbital angular momentum component: $\Omega=\Sigma+\Lambda$. We will only consider the lowest spin orbit state: $\bar{\Omega}=|\Omega|=\frac{1}{2}$. This implies that we will only consider spin orbit conserving transitions. The spin orbit conserving transitions are determined by the $V_{\text {sum }}$ potential energy surface ${ }^{31}$, so that is the PES that we will use, see appendix A. For a ${ }^{2} \Pi$ molecule the rotational wave function is that of a symmetric top. Its eigen functions are given by

$$
\Psi_{j m \Omega}\left(\gamma_{a}, \phi_{a}\right)=\sqrt{\frac{2 j+1}{4 \pi}} D_{m \Omega}^{* j}\left(\gamma_{a}, \phi_{a}, 0\right)
$$

Because of the $\Lambda$-doublet splitting in NO a more exact basis is given by

$$
\Psi_{j m \bar{\Omega} \epsilon}=\frac{1}{\sqrt{2}}\left(\Psi_{j m \bar{\Omega}}+\epsilon \Psi_{j m-\bar{\Omega}}\right)
$$

These states have a definite parity $p=(-1)^{j-\epsilon / 2}$, where $\epsilon$ is the symmetry index. This symmetry index can have the values $\epsilon=1$ (e) and $\epsilon=-1$ (f). As said before, the angular momentum component along the apse is conserved, $m_{a}^{\prime}=m_{a}$, and spin orbit breaking transitions are ignored, $\Omega^{\prime}=\Omega$. For the ground state of NO $j=\frac{1}{2}, \bar{\Omega}=\frac{1}{2}$, and $\epsilon=-1$. The product of the rotational wave functions is then, from appendix D:

$$
\Psi_{j^{\prime} m_{a} \bar{\Omega} \epsilon^{\prime}}^{*} \Psi_{\frac{1}{2} m_{a} \bar{\Omega} \epsilon}=\frac{\sqrt{2 j^{\prime}+1}}{4 \pi} \frac{1}{\sqrt{2}} P_{j^{\prime}-\frac{\epsilon^{\prime} \epsilon}{2}}\left(\cos \gamma_{a}\right)
$$

For symmetry index breaking transitions the sign of this expression should be equal to the sign of $m_{a}$, see equation (D12), but we here ignore this since it does not affect the differential cross-section. From equation (35) it follows that we have a $P_{\Delta j}$ for symmetry index conserving transitions and a $P_{\Delta j+1}$ for symmetry index breaking transitions. The differential cross sections for the symmetry index breaking transitions to $j^{\prime}$ are therefore similar to those for the symmetry index conserving transitions to $j^{\prime}+1$. Because of this similarity we will label the differential cross sections with $n=j^{\prime}-\frac{\epsilon^{\prime} \epsilon}{2}$, the "index" of the Legendre polynomial in equation (35). We will call $n$ the parity pair number, since these similar differential cross sections come in pairs $\left(\left(j^{\prime}, \epsilon^{\prime}\right)\right.$ and $\left.\left(j^{\prime}-\epsilon^{\prime} \epsilon,-\epsilon^{\prime}\right)\right)$ and their final states have the same parity $p=(-1)^{j-\epsilon / 2}$. In the apse frame the scattering amplitude becomes (after performing the trivial integration over $\phi_{a}$ ):

$$
g_{j^{\prime} \epsilon^{\prime}}(\beta)=C(\beta) \frac{\sqrt{2 j^{\prime}+1}}{2} \frac{1}{\sqrt{2}} \int_{0}^{\pi} g_{\text {geom }}\left(\gamma_{a} ; \beta\right) e^{i \eta_{j^{\prime}}\left(\gamma_{a} ; \beta\right)} P_{j^{\prime}-\frac{\epsilon^{\prime} \epsilon}{2}}\left(\cos \gamma_{a}\right) \sin \gamma_{a} d \gamma_{a}
$$


The phase shift $\eta_{j^{\prime} \epsilon^{\prime}}\left(\gamma_{a} ; \beta\right)$ is equal to $\eta_{j^{\prime}}\left(\gamma_{a} ; \beta\right)$, since it only depends on the energy levels and the symmetry index has only a very small effect on those levels, see also appendix B. Note the similarity between equations (36) and (31), the symmetry index conserving transitions of the ${ }^{2} \Pi$ case correspond to the transitions of the ${ }^{1} \Sigma$ case for the same $j^{\prime}$. The differential cross-section (now assuming constant curvature) is given by

$$
\frac{d \sigma_{\frac{1}{2}, \epsilon \rightarrow j^{\prime}, \epsilon^{\prime}}}{d \omega_{a}}(\beta)=\frac{d \sigma^{\text {geom }}}{d \omega_{a}}(\beta) \cdot \frac{\frac{\left(2 j^{\prime}+1\right)}{8}\left|\int_{0}^{\pi} e^{i \eta_{j^{\prime}}\left(\gamma_{a} ; \beta\right)} P_{j^{\prime}-\frac{\epsilon^{\prime} \epsilon}{2}}\left(\cos \gamma_{a}\right) \sin \gamma_{a} d \gamma_{a}\right|^{2}}{\sum_{\text {all } j^{\prime}, \epsilon^{\prime}} \frac{\left(2 j^{\prime}+1\right)}{8}\left|\int_{0}^{\pi} e^{i \eta_{j^{\prime}}\left(\gamma_{a} ; \beta\right)} P_{j^{\prime}-\frac{\epsilon^{\prime} \epsilon}{2}}\left(\cos \gamma_{a}\right) \sin \gamma_{a} d \gamma_{a}\right|^{2}}
$$

In appendix $\mathrm{E}$ it is shown that the sum over all final states in the denominator of equation (37) is equal to one if $\Delta E_{\mathrm{rot}}(\Delta j)=0$ for all $\Delta j$. That means that the expression for the scattering amplitude does not need any normalisation factors in that case, just as we saw for Ne-CO.

[Figure 8 about here.]

[Figure 9 about here.]

[Figure 10 about here.]

The potential energy surface for He-NO is plotted in figure 8. The format of the figure is the same as that for the CO hardshell presented earlier. The same is true for the phase shift plotted in figure 9. Comparing the two cases, the most important difference is the difference in magnitude of the phase shift at the not-O-end. For CO it is almost twice as large as for NO. The effects can be seen in the differential cross-sections, figures 6 and 10: for NO there is for most rotational transitions not enough phase shift to show more than one maximum, whereas for CO there are several maxima in most of the differential cross-sections. In figure 10 the differential cross sections for He-NO as calculated by QQT are compared to close coupling calculations done by $\mathrm{Kłos}^{35}$ using the full potential energy surface and the computer code HIBRIDON ${ }^{36}$. For the first few parity pair numbers the effect of neglecting the diffraction contribution is clearly seen: the QQT calculations miss the oscillatory structures. At higher parity pair numbers the results from the QQT calculations are (much) larger. This could 


\section{FINAL REMARKS}

Although diffraction effects are not included, and the potential energy surface is represented by a hardshell potential, the QQT is still able to reproduce the basic structure of the differential cross-sections. Moreover, this structure is easily related to the given anisotropy of the considered potential energy surfaces. Using the constant curvature approximation has hardly any influence on the accuracy of the differential cross sections as calculated by the QQT. This makes the reasoning about the physics in these collision processes even more simple; one only has to consider the phase shifts originating from the anisotropy of the potential energy surface. The new results on the normalisation factor emphasise its physical basis: the QQT provides a straightforward, physical derivation for diffractionless scattering from a hardshell with vanishing rotational energy level spacings. To account for non-vanishing 
rotational energy level spacings, this QQT result is then extended using a normalisation procedure to preserve its self-consistency.

\section{Acknowledgments}

The authors would like to express their gratitude to dr. G.C. McBane for providing them with the differential cross-sections for Ne-CO from his close coupling calculations and the turning points for the Ne-CO potential at $511 \mathrm{~cm}^{-1}$.

\section{Appendix A: HARDSHELLS}

The hardshell for NO colliding with He is approximated using the $514 \mathrm{~cm}^{-1}$ equipotential surface from the $V_{\text {sum }}$ PES calculated by $\mathrm{Kłos}^{37}$. For the Ne-CO collisions we used the $511 \mathrm{~cm}^{-1}$ contour of the CCSD(T) surface S2 from McBane and Cybulski ${ }^{3}$ as provided by McBane. The $460 \mathrm{~cm}^{-1}$ contour as given in Lorenz et al. ${ }^{4}$ gives almost the same results. These surfaces were fitted to a legendre expansion

$$
R_{\mathrm{S}}\left(\gamma_{R}\right)=\sum_{n=0}^{n_{\max }} c_{n} P_{n}\left(\cos \gamma_{R}\right)
$$

The coefficients $c_{n}$ for the two hardshells are given in table I.

[Table 1 about here.]

\section{Appendix B: ENERGY LEVELS}

For CO the rotational energy levels are given by (see Zare ${ }^{38}$, eq. 6, p.299)

$$
E(j)=B_{0}(j(j+1))
$$

with $B_{0}=1.9225 \mathrm{~cm}^{-1}{ }^{39}$

For NO, in the lower spin-orbit state $\left(\bar{\Omega}=\frac{1}{2}\right)$, the rotational energy levels are given by (see Zare, eq. 30, p.303)

$$
E(j)=B_{0}\left[\left(j-\frac{1}{2}\right)\left(j+\frac{3}{2}\right)-\frac{1}{2} \sqrt{4\left(j+\frac{1}{2}\right)^{2}+Y(Y-4)}\right]
$$

with $Y=A_{0} / B_{0}$, and $A_{0}=123.13 \mathrm{~cm}^{-1}$, and $B_{0}=1.6961 \mathrm{~cm}^{-1} \cdot{ }^{40}$ 


\section{Appendix C: THE GEOMETRICAL DIFFERENTIAL CROSS-SECTION}

The geometrical cross-section of a hardshell is the amount of area presented by that hardshell to the incoming beam. If we consider all surface elements of the hardshell separately, we get the geometrical differential cross-section. It is the ratio between the infinitesimal area presented by a surface element to the incoming beam and the infinitesimal solid angle spanned by the normal vectors of this surface element, see the derivation below. By defining this geometrical differential cross-section in the apse frame, it is completely determined by the geometry of the hardshell. To transform it to the standard frame one has to include the Jacobian $d \omega_{a} / d \omega$, but that Jacobian depends on the inelasticity of the collision. The expression for this geometrical differential cross-section is derived in the following paragraphs.

\section{[Figure 13 about here.]}

First we show why the relation between $d \sigma$ and $d \omega_{a}$ involves the curvature of the hardshell, and then we show how to calculate that curvature. See figure 13. The infinitesimal incoming beam with cross-sectional area $d \sigma$ and incoming wave vector $\boldsymbol{k}$ hits the hardshell on the surface element $d A$ that has a normal $\hat{\boldsymbol{n}}$. Then

$$
d \sigma=|\hat{\boldsymbol{k}} \cdot \hat{\boldsymbol{n}}| d A=|\cos \beta| d A
$$

Different parts of the surface element $d A$ have a slightly different direction of their normal vector. All those normal vectors together span an element of solid angle $d \omega_{a}=\sin \beta d \alpha d \beta$. For a sphere the relation between the surface element $d A$ and the element of solid angle $d \omega_{a}$ follows from the definition of a solid angle (on the unit sphere a solid angle is equal to the surface area it encloses):

$$
d \omega_{a}=d \beta \cdot \sin \beta d \alpha=\frac{R d \beta \cdot R \sin \beta d \alpha}{R R}=\frac{d A}{R^{2}}
$$

For a general convex surface we realise that $d \beta$ and $\sin \beta d \alpha$ form the sides of a rectangle on the unit sphere. Multiplying those sides with the local radii of curvature $\rho_{1}$ and $\rho_{2}$ of the surface, as we did for the case of a sphere, we get the area of the enclosed surface element:

$$
d \omega_{a}=d \beta \cdot \sin \beta d \alpha=\frac{\rho_{1} d \beta \cdot \rho_{2} \sin \beta d \alpha}{\rho_{1} \rho_{2}}=\frac{d A}{\rho_{1} \rho_{2}}
$$


The molecule fixed geometrical differential cross-section is therefore in general given by

$$
\frac{d \sigma^{\text {geom }}}{d \omega_{a}}\left(\gamma_{a} ; \beta\right)=|\cos \beta| \rho_{1}\left(\gamma_{a}\right) \rho_{2}\left(\gamma_{a}\right)
$$

and for a sphere by

$$
\frac{d \sigma^{\text {geom }}}{d \omega_{a}}\left(\gamma_{a} ; \beta\right)=|\cos \beta| R^{2}
$$

The radii of curvature $\rho_{1}$ and $\rho_{2}$ are calculated as

$$
\begin{aligned}
\rho_{1} & =\frac{d s_{1}}{d \gamma_{a}} \\
\rho_{2} & =\frac{R_{\mathrm{S}}\left(\gamma_{R}\right) \sin \left(\gamma_{R}\right)}{\sin \left(\gamma_{a}\right)}
\end{aligned}
$$

$d s_{1} / d \gamma_{a}$ is the $\gamma_{a}$ derivative of the arc length of the hardshell in a plane containing the molecular axis. The arc length is given by $d s_{1}=\sqrt{d z_{\text {cyl }}^{2}+d r_{\text {cyl }}^{2}}$, using the cylindrical coordinates defined in equations (9) and (10), and $d \gamma_{a}$ is the angle over which the normal has rotated while travelling the piece of arc length $d s_{1}$. It is instructive to apply equation (C6) to the case that the curve $s_{1}$ forms a circle. The second radius of curvature $\rho_{2}$ can be calculated explicitly because of cylinder symmetry and is given by the distance from the molecular axis to the hardshell along the surface normal, see also Bronshtein and Semendyayev ${ }^{41,42}$.

\section{Appendix D: DERIVATION OF $\Psi_{f}^{*} \Psi_{i}$ FOR THE CASE OF NO}

To calculate $\Psi_{j^{\prime} m \bar{\Omega} \epsilon^{\prime}}^{*} \Psi_{j m \bar{\Omega} \epsilon}$ we first calculate $\Psi_{j^{\prime} m \Omega}^{*} \Psi_{j m \Omega}$. Using Zare ${ }^{38}$ eq. 1, p. 297, and $m^{\prime}=m, \Omega^{\prime}=\Omega$

$$
\Psi_{j^{\prime} m \Omega}^{*} \Psi_{j m \Omega}=\sqrt{\frac{2 j^{\prime}+1}{4 \pi}} D_{m \Omega}^{j^{\prime}} \sqrt{\frac{2 j+1}{4 \pi}} D_{m \Omega}^{* j}
$$

Using Zare equation 3.125, page 105

$$
D_{m \Omega}^{* j}=D_{-m-\Omega}^{j}(-1)^{m-\Omega}
$$

Using Zare equation 3.105, page 99 ( $D_{00}^{j_{3}}$ is real)

$$
D_{m \Omega}^{j^{\prime}} D_{-m-\Omega}^{j}(-1)^{m-\Omega}=(-1)^{m-\Omega} \sum_{j_{3}}\left\langle\begin{array}{cc|c}
j^{\prime} & j & j_{3} \\
m & -m & 0
\end{array}\right\rangle\left\langle\begin{array}{cc|c}
j^{\prime} & j & j_{3} \\
\Omega & -\Omega & 0
\end{array}\right\rangle D_{00}^{j_{3}}
$$


The expressions between the brackets are the Clebsch-Gordan coefficients $\left\langle j_{1}, m_{1}, j_{2}, m_{2} \mid j_{3}, m_{3}\right\rangle$. We need the following symmetry property of the Clebsch-Gordan coefficients, see Zare equation 2.26 on page 49

$$
\left\langle\begin{array}{cc|c}
j_{1} & j_{2} & j_{3} \\
m_{1} & m_{2} & m_{3}
\end{array}\right\rangle=(-1)^{j_{1}+j_{2}-j_{3}}\left\langle\begin{array}{cc|c}
j_{1} & j_{2} & j_{3} \\
-m_{1} & -m_{2} & -m_{3}
\end{array}\right\rangle
$$

Using this property and the fact that $D_{00}^{j_{3}}=P_{j_{3}}$ (Zare equation 3.95 on page 97 )

$$
\begin{aligned}
& D_{m \Omega}^{j^{\prime}} D_{-m-\Omega}^{j}(-1)^{m-\Omega}= \\
& (-1)^{m-\Omega} \sum_{j_{3}}\left(\frac{m}{|m|}\right)^{j^{\prime}+j-j_{3}}\left\langle\begin{array}{cc|c}
j^{\prime} & j & j_{3} \\
|m| & -|m| & 0
\end{array}\right)\left(\frac{\Omega}{|\Omega|}\right)^{j^{\prime}+j-j_{3}}\left\langle\begin{array}{cc|c}
j^{\prime} & j & j_{3} \\
|\Omega| & -|\Omega| & 0
\end{array}\right\rangle P_{j_{3}}
\end{aligned}
$$

For our NO case $j=\frac{1}{2},|m|=|\Omega|=\frac{1}{2}$, and therefore $j_{3}=j^{\prime} \pm \frac{1}{2}$

$$
\begin{aligned}
& D_{m \Omega}^{j^{\prime}} D_{-m-\Omega}^{\frac{1}{2}}(-1)^{m-\Omega}= \\
& (-1)^{m-\Omega}\left[\left(\frac{m \Omega}{|m \Omega|}\right)^{1}\left\langle\begin{array}{cc|c}
j^{\prime} & \frac{1}{2} & j^{\prime}-\frac{1}{2} \\
\frac{1}{2} & -\frac{1}{2} & 0
\end{array}\right\rangle^{2} P_{j^{\prime}-\frac{1}{2}}+\left(\frac{m \Omega}{|m \Omega|}\right)^{0}\left\langle\begin{array}{cc|c}
j^{\prime} & \frac{1}{2} \\
\frac{1}{2} & -\frac{1}{2} & j^{\prime}+\frac{1}{2} \\
0
\end{array}\right\rangle^{2} P_{j^{\prime}+\frac{1}{2}}\right]
\end{aligned}
$$

Table 2.4 on page 57 from Zare gives a value of $\frac{1}{2}$ for the square of both of the above ClebschGordan coefficients. The factor $(-1)^{m-\Omega}$ coming from equation (D2) is equal to $\frac{m \Omega}{|m \Omega|}$ for $|m|=|\Omega|=\frac{1}{2}$. So

$$
D_{m \Omega}^{j^{\prime}} D_{-m-\Omega}^{\frac{1}{2}}(-1)^{m-\Omega}=\frac{1}{2}\left[P_{j^{\prime}-\frac{1}{2}}+\frac{m \Omega}{|m \Omega|} P_{j^{\prime}+\frac{1}{2}}\right]
$$

And

$$
\Psi_{j^{\prime} m \Omega}^{*} \Psi_{\frac{1}{2} m \Omega}=\frac{\sqrt{2 j^{\prime}+1}}{4 \pi} \frac{1}{\sqrt{2}}\left[P_{j^{\prime}-\frac{1}{2}}+\frac{m \Omega}{|m \Omega|} P_{j^{\prime}+\frac{1}{2}}\right]
$$

Now we will write $\Psi_{j^{\prime} m \bar{\Omega} \epsilon^{\prime}}^{*} \Psi_{j m \bar{\Omega} \epsilon}$ in terms of $\Psi_{j^{\prime} m \Omega}^{*} \Psi_{j m \Omega}$. First

$$
\Psi_{j m \bar{\Omega} \epsilon}=\frac{1}{\sqrt{2}}\left(\Psi_{j m \bar{\Omega}}+\epsilon \Psi_{j m-\bar{\Omega}}\right)
$$

Ignoring spin-orbit changing transitions

$$
\Psi_{j^{\prime} m \bar{\Omega} \epsilon^{\prime}}^{*} \Psi_{j m \bar{\Omega} \epsilon}=\frac{1}{2}\left[\Psi_{j^{\prime} m \bar{\Omega}}^{*} \Psi_{j m \bar{\Omega}}+\epsilon^{\prime} \epsilon \Psi_{j^{\prime} m-\bar{\Omega}}^{*} \Psi_{j m-\bar{\Omega}}\right]
$$


Using equation (D8)

$$
\Psi_{j^{\prime} m \bar{\Omega} \epsilon^{\prime}}^{*} \Psi_{\frac{1}{2} m \bar{\Omega} \epsilon}=\frac{1}{2} \frac{\sqrt{2 j^{\prime}+1}}{4 \pi} \frac{1}{\sqrt{2}}\left[\left(1+\epsilon^{\prime} \epsilon\right) P_{j^{\prime}-\frac{1}{2}}+\left(1-\epsilon^{\prime} \epsilon\right) \frac{m}{|m|} P_{j^{\prime}+\frac{1}{2}}\right]
$$

Or

$$
\Psi_{j^{\prime} m \bar{\Omega} \epsilon^{\prime}}^{*} \Psi_{\frac{1}{2} m \bar{\Omega} \epsilon}= \begin{cases}\frac{\sqrt{2 j^{\prime}+1}}{4 \pi} \frac{1}{\sqrt{2}} P_{j^{\prime}-\frac{1}{2}} & \epsilon^{\prime} \epsilon=+1 \\ \frac{\sqrt{2 j^{\prime}+1}}{4 \pi} \frac{1}{\sqrt{2}} \frac{m}{|m|} P_{j^{\prime}+\frac{1}{2}} & \epsilon^{\prime} \epsilon=-1\end{cases}
$$

The sign $\frac{m}{|m|}$ will cancel when calculating the differential cross-section. This allows us to write

$$
\Psi_{j^{\prime} m \bar{\Omega} \epsilon^{\prime}}^{*} \Psi_{\frac{1}{2} m \bar{\Omega} \epsilon}=\frac{\sqrt{2 j^{\prime}+1}}{4 \pi} \frac{1}{\sqrt{2}} P_{j^{\prime}-\frac{\epsilon^{\prime} \epsilon}{2}}
$$

\section{Appendix E: NORMALISATION OF THE DIFFERENTIAL CROSS-SECTION}

In sections III and IV it was announced that the denominator in equations (32) and (37) will be equal to one when $\Delta E_{\text {rot }}(\Delta j)=0$ for all $\Delta j$. In this appendix we will give the derivation.

For the ${ }^{1} \Sigma$ case we start with the denominator in equation (32), using $j^{\prime}=\Delta j$ :

$$
\sum_{\Delta j=0}^{\infty} \frac{(2 \Delta j+1)}{4}\left|\int_{0}^{\pi} e^{i \eta_{\Delta j}\left(\gamma_{a} ; \beta\right)} P_{\Delta j}\left(\cos \gamma_{a}\right) \sin \gamma_{a} d \gamma_{a}\right|^{2}
$$

Introducing orthonormal $\tilde{P}_{n}=\sqrt{\frac{2 n+1}{2}} P_{n}$ such that $\int_{0}^{\pi} \tilde{P}_{m} \tilde{P}_{n} \sin \gamma_{a} d \gamma_{a}=\delta_{m n}$ the above is equal to

$$
\sum_{\Delta j=0}^{\infty} \frac{1}{2}\left|\int_{0}^{\pi} e^{i \eta_{\Delta j}\left(\gamma_{a} ; \beta\right)} \tilde{P}_{\Delta j}\left(\cos \gamma_{a}\right) \sin \gamma_{a} d \gamma_{a}\right|^{2}
$$

Expanding the phase shift factor in terms of the orthonormal Legendre polynomials, $e^{i \eta_{\Delta j}\left(\gamma_{a} ; \beta\right)}=\sum_{n}^{\infty} a_{n} \tilde{P}_{n}\left(\cos \gamma_{a}\right)$, we get

$$
\begin{gathered}
\sum_{\Delta j=0}^{\infty} \frac{1}{2}\left|\int_{0}^{\pi} \sum_{n}^{\infty} a_{n} \tilde{P}_{n}\left(\cos \gamma_{a}\right) \tilde{P}_{\Delta j}\left(\cos \gamma_{a}\right) \sin \gamma_{a} d \gamma_{a}\right|^{2} \\
=\frac{1}{2} \sum_{\Delta j=0}^{\infty}\left|\sum_{n}^{\infty} a_{n} \delta_{n, \Delta j}\right|^{2}=\frac{1}{2} \sum_{\Delta j=0}^{\infty}\left|a_{\Delta j}\right|^{2}
\end{gathered}
$$

If we assume $\Delta E_{\mathrm{rot}}(\Delta j)=0$ then the phase shift factor no longer depends on $\Delta j$ and the above sum is equal to

$$
\frac{1}{2} \int_{0}^{\pi}\left|e^{i \eta\left(\gamma_{a} ; \beta\right)}\right|^{2} \sin \gamma_{a} d \gamma_{a}=1
$$


For the ${ }^{2} \Pi$ case we start with the denominator in equation (37), using $j^{\prime}-\frac{1}{2}=\Delta j$ :

$$
\sum_{\Delta j=0}^{\infty} \frac{(2 \Delta j+2)}{8}\left\{\left|\int_{0}^{\pi} e^{i \eta_{\Delta j}\left(\gamma_{a} ; \beta\right)} P_{\Delta j}\left(\cos \gamma_{a}\right) \sin \gamma_{a} d \gamma_{a}\right|^{2}+\left|\int_{0}^{\pi} e^{i \eta_{\Delta j}\left(\gamma_{a} ; \beta\right)} P_{\Delta j+1}\left(\cos \gamma_{a}\right) \sin \gamma_{a} d \gamma_{a}\right|^{2}\right\}
$$

Rewriting the sum over $\Delta j$ we get

$$
\begin{gathered}
\sum_{\Delta j=0}^{\infty} \frac{(2 \Delta j+2)}{8}\left|\int_{0}^{\pi} e^{i \eta_{\Delta j}\left(\gamma_{a} ; \beta\right)} P_{\Delta j}\left(\cos \gamma_{a}\right) \sin \gamma_{a} d \gamma_{a}\right|^{2}+\sum_{\Delta j=1}^{\infty} \frac{2 \Delta j}{8}\left|\int_{0}^{\pi} e^{i \eta_{\Delta j}\left(\gamma_{a} ; \beta\right)} P_{\Delta j}\left(\cos \gamma_{a}\right) \sin \gamma_{a} d \gamma_{a}\right|^{2} \\
=\sum_{\Delta j=0}^{\infty} \frac{(2 \Delta j+1)}{4}\left|\int_{0}^{\pi} e^{i \eta_{\Delta j}\left(\gamma_{a} ; \beta\right)} P_{\Delta j}\left(\cos \gamma_{a}\right) \sin \gamma_{a} d \gamma_{a}\right|^{2}
\end{gathered}
$$

This is equal to equation (E1) and is therefore equal to one under the same assumptions and for the same reasons.

If we do not use the constant curvature approximation, equation (E5) will be replaced by

$$
\frac{1}{2} \int_{0}^{\pi}\left|\frac{g_{\text {geom }}\left(\gamma_{a} ; \beta\right)}{k R_{\text {eff }} \sqrt{|\cos \beta|}} e^{i \eta\left(\gamma_{a} ; \beta\right)}\right|^{2} \sin \gamma_{a} d \gamma_{a}=1
$$

See equations (15), (21), and (22).

1 A. Gijsbertsen, H. Linnartz, S. Stolte, and C. A. Taatjes, J. Am. Chem. Soc. 128, 8777 (2006).

2 S. Antonova, A. Lin, A. P. Tsakotellis, and G. C. McBane, J. Chem. Phys 110, 11742 (1999).

3 G. C. McBane and S. M. Cybulski, J. Chem. Phys. 110, 11734 (1999).

${ }^{4}$ K. T. Lorenz, D. W. Chandler, and G. C. McBane, J. Phys. Chem. A. 106, 1144 (2002).

5 D. A. Hostutler, T. C. Smith, G. D. Hager, G. C. McBane, and M. C. Heaven, J. Chem. Phys 120, $7483(2004)$.

${ }^{6}$ G. A. Amaral, F. J. Aoiz, L. Banares, J. Barr, V. J. Herrero, B. Martinez-Haya, M. Menendez, G. A. Pino, I. Tanarro, I. Torres, et al., J. Phys. Chem. A 109, 9402 (2005).

7 H. Joswig, P. Andresen, and R. Schinke, J. Chem. Phys. 85, 1904 (1986).

8 H. Meyer, J. Chem. Phys. 102, 3151 (1995).

9 M. Yang and M. H. Alexander, J. Chem. Phys. 103, 6973 (1995).

10 M. Drabbels and A. M. Wodtke, J. Chem. Phys. 106, 3024 (1997). 
11 M. H. Alexander, Faraday Discuss. Chem. Soc. 113, 437 (1999).

12 J. Kłos, G. Chalasinski, M. T. Berry, R. A. Kendall, R. Burcl, M. Szczesniak, and S. M. Cybulski, J. Chem. Phys. 112, 4952 (2000).

13 M. S. Westley, K. T. Lorenz, D. W. Chandler, and P. L. Houston, J. Chem. Phys. 114, 2669 (2001).

14 P. A. Barrass, P. Sharkey, and I. W. M. Smith, Phys. Chem. Chem. Phys. 5, 1400 (2003).

15 Y. Kim, H. Meyer, and M. H. Alexander, J. Chem. Phys. 121, 1339 (2004).

16 M. J. L. de Lange, S. Stolte, C. A. Taatjes, J. Kłos, G. C. Groenenboom, and A. v.d. Avoird, J. Chem. Phys. 121, 11691 (2004).

17 A. Gijsbertsen, H. Linnartz, J. Kłos, and S. Stolte, Physica Scripta 72, 1 (2005).

18 A. Gijsbertsen, H. Linnartz, and S. Stolte (2006).

19 C. A. Taatjes, A. Gijsbertsen, M. J. L. de Lange, and S. Stolte, J. Phys. Chem. A 111, 7631 (2007).

20 J. Klos, F. J. Aoiz, J. E. Verdasco, M. Brouard, S. Marinakis, and S. Stolte, J. Chem. Phys. 127 (2007).

21 D. K. Hoffman, J. Chem. Phys 50, 4823 (1969).

22 G. T. Evans, R. S. C. She, and R. B. Bernstein, J. Chem. Phys. 82, 2258 (1985).

23 M. Esposito and G. T. Evans, J. Chem. Phys. 97, 4846 (1992).

24 V. Khare, D. J. Kouri, and D. K. Hoffmann, J. Chem. Phys. 74, 2275 (1981).

25 V. Khare, D. J. Kouri, and D. K. Hoffmann, J. Chem. Phys. 76, 4493 (1982).

26 J. L. van Leuken, J. Bulthuis, S. Stolte, and J. G. Snijders, Chem. Phys. Lett. 260, 595 (1996).

27 M. J. L. de Lange, M. Drabbels, P. T. Griffiths, J. Bulthuis, and J. G. Snijders, Chem. Phys. Lett. 313, 491 (1999).

28 M. S. Child, Molecular Collision Theory (Dover Mineola NY, 1996).

29 U. Beck, U. Ross, and W. Schlepper, Z. Physik A 293, 107 (1979).

30 A. M. Arthurs and A. Dalgarno, Proc. Roy. Soc. (London), Ser. A 256, 540 (1960).

31 M. H. Alexander, J. Chem. Phys. 99, 7725 (1993).

32 J. M. Hutson and S. Green, MOLSCAT computer code (distributed by Collaborative Computational Project No. 6 of the Engineering and Physical Sciences Research Council, UK, 1994), 
version 14 .

33 F. J. Aoiz, J. E. Verdasco, V. J. Herrero, V. S. Rábanos, and M. H. Alexander, J. Chem. Phys. 119, $5860(2003)$.

34 J. Klos, F. J. Aoiz, J. E. Verdasco, M. Brouard, S. Marinakis, and S. Stolte, J. Chem. Phys. 127 (2007).

35 A. Gijsbertsen, H. Linnartz, G. Rus, A. E. Wiskerke, S. Stolte, D. W. Chandler, and J. Kłos, J. Chem. Phys. 123, 224305 (2005).

36 HIBRIDON is a package of programs for the time independent quantum treatment of inelastic collisions written by M. H. Alexander, D. E. Manolopoulos, H.J. Werner, and B. Follmeg, with contributions by P. F. Vohralik, D. Lemoine, G. Corey, R. Gordon, B. Johnson, T. Orlikowski, A. Berning, A. Degli-Esposti, C. Rist, P. Dagdigian, B. Pouilly, G. van der Sanden, M. Yang, F. de Weerd, and S. Gregurick. More information and a copy of the code can be obtained from http://www.chem.umd.edu/physical/alexander/hibridon/.

37 J. Kłos, G. Chalasinski, M. T. Berry, R. Bukowski, and S. M. Cybulski, J. Chem. Phys. 112, $2195(2000)$.

38 R. N. Zare, Angular Momentum: Understanding spatial aspects in chemisty and physics (John Wiley \& Sons, New York, 1988).

39 J. Brown and A. Carrington, Rotational Spectroscopy of Diatomic Molecules (Cambridge University Press, 2003).

40 C. Amiot, J. Molec. Spectrosc. 94, 150 (1982).

41 I. N. Bronstein, K. A. Semendjajew, G. Grosche, V. Ziegler, D. Ziegler, and E. Zeidler, TeubnerTaschenbuch der Mathematik (Teubner, 1995).

42 I. N. Bronshtein and K. A. Semendyayev, Handbook of Mathematics (Springer, 1985).

43 M. H. Alexander and S. Stolte, J. Chem. Phys. 112, 8017 (2000).

${ }^{44}$ In e.g. Child ${ }^{28}$ Ch. 6: $d \sigma / d \omega=k^{\prime} / k \cdot|f|^{2}$. But instead Alexander and Stolte ${ }^{43}$ use a dimensionless scattering amplitude: instead of $f e^{i k r} / r$ they write $\sqrt{k k^{\prime}} f e^{i k r} / \sqrt{k k^{\prime}} r$ and introduce the dimensionless scattering ampitude $\tilde{f}=\sqrt{k k^{\prime}} f$ that gives $d \sigma / d \omega=k^{\prime} / k \cdot|f|^{2}=1 / k^{2} \cdot|\tilde{f}|^{2}$. We will use $f=\tilde{f}$.

45 The difference between using $R_{\text {eff }}$ or $R_{0}$ is best illustrated by considering a flat circular disk 
with an isotropic directional distribution. Here $R_{0}$ is equal to zero and $R_{\text {eff }}$ is equal to the radius of the disk. For the applications considered in this paper the difference between the two radii is approximately $5 \%$. 
Table I: Hardshell Legendre polynomial approximation coefficients for use in Eq. (A1).

\begin{tabular}{|c|c|c|}
\hline$n$ & $c_{n}$ for He-NO hardshell $\left[a_{0}\right]$ & $c_{n}$ for Ne-CO hardshell $\left[a_{0}\right]$ \\
\hline \hline 0 & 4.8637 & 5.2822 \\
1 & 0.1983 & 0.3665 \\
2 & 0.6908 & 0.7218 \\
3 & -0.0126 & 0.0504 \\
4 & -0.1497 & -0.0685 \\
5 & -0.0012 & -0.0284 \\
6 & 0.0263 & 0.0048 \\
7 & - & 0.0080 \\
\hline
\end{tabular}




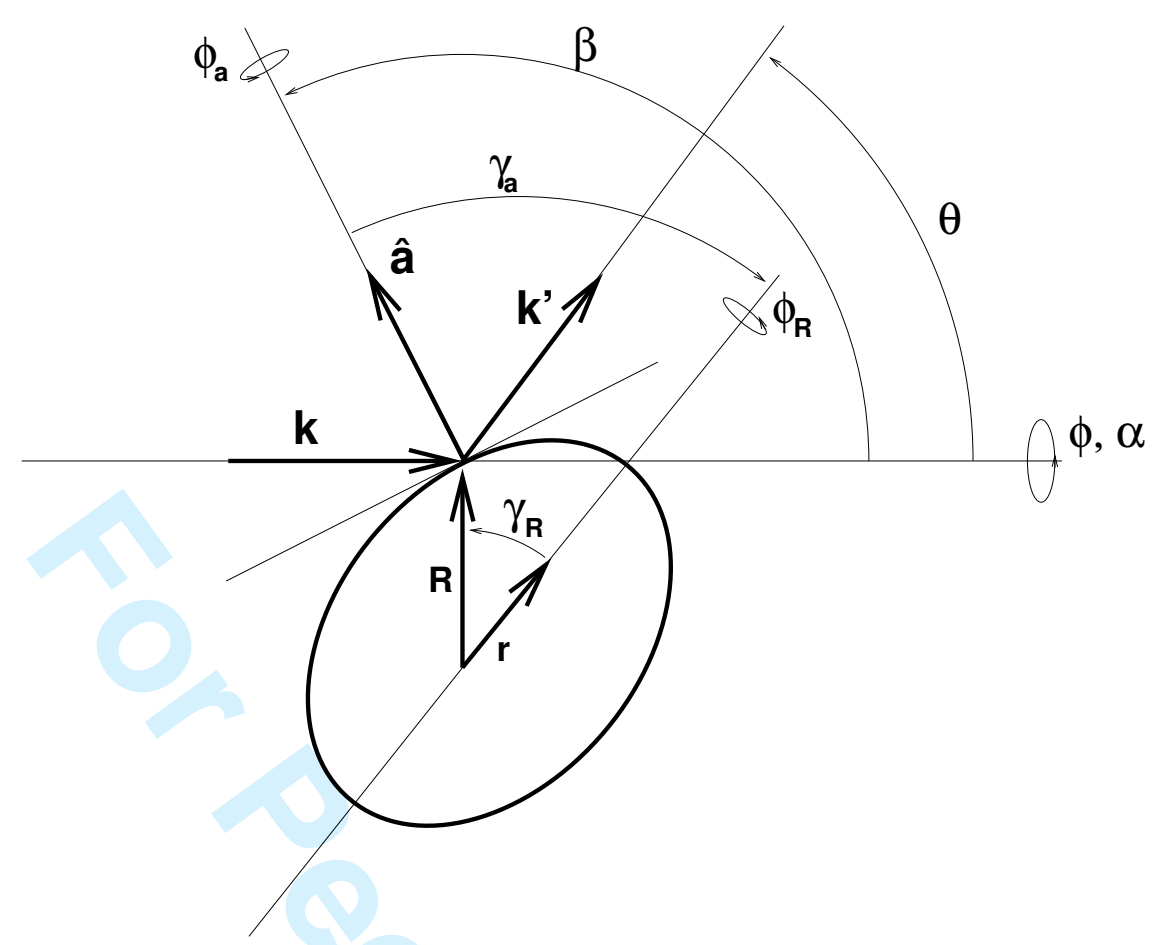

Figure 1: All relevant vectors and their angles. The azimuthal angles are indicated with a small ellipse on their axis. To clarify their meaning most of this figure is repeated twice in figure 2: once for the case that the vectors $\boldsymbol{r}$ and $\boldsymbol{R}$ have an azimuthal angle $\phi_{a}$ of zero with respect to the apse and once for the case that that angle is $\pi$. The centre of mass of the molecule is at the starting point of the vector $\boldsymbol{R}$. The outgoing wave vector $\boldsymbol{k}^{\prime}$ as drawn corresponds to the elastic scattering case. 

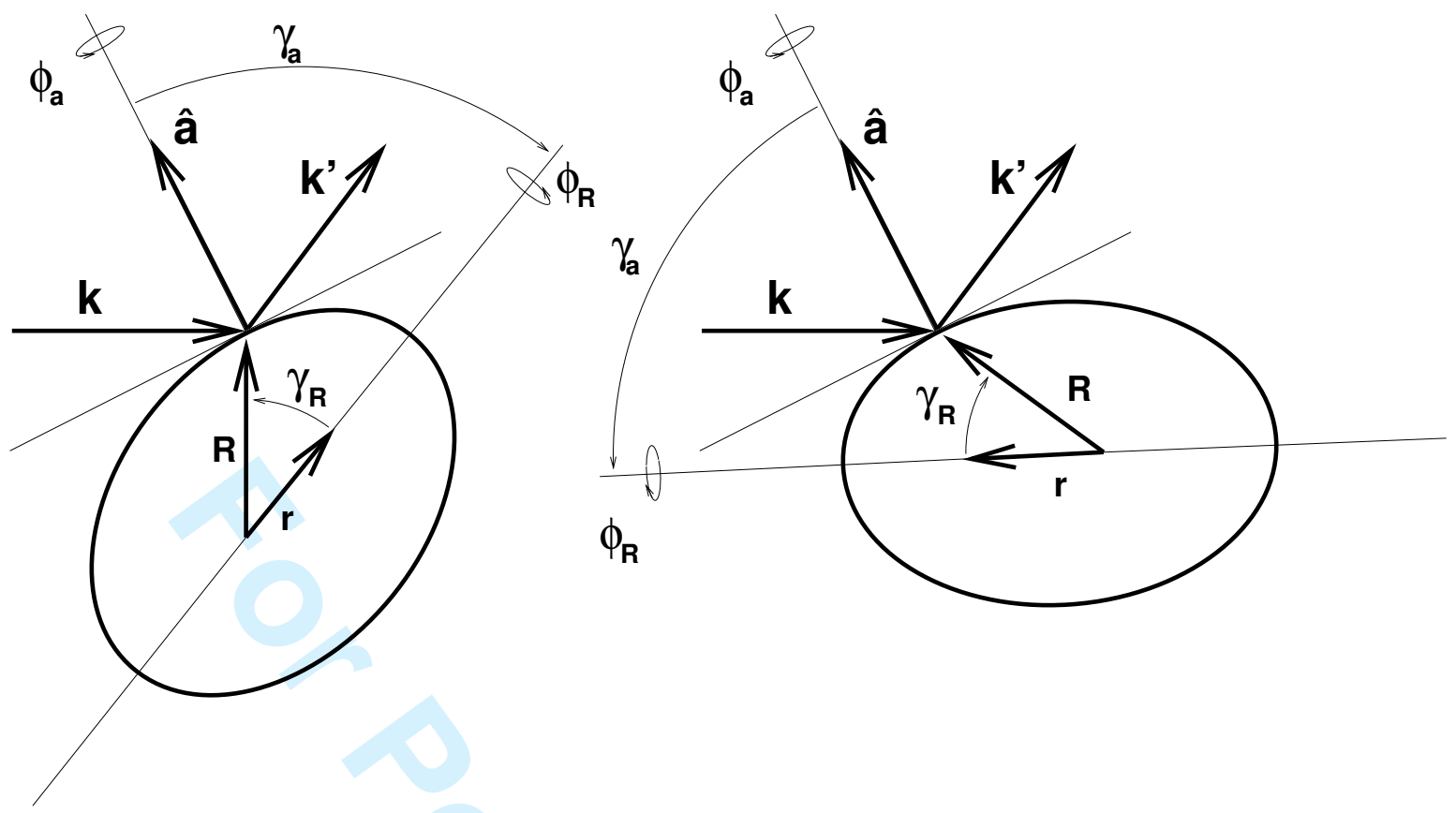

Figure 2: The meaning of the azimuthal angle $\phi_{a}$. On the right side the vectors $\boldsymbol{r}$ and $\boldsymbol{R}$ have an azimuthal angle $\phi_{a}$ of zero with respect to the apse and on the left side that angle is $\pi$.

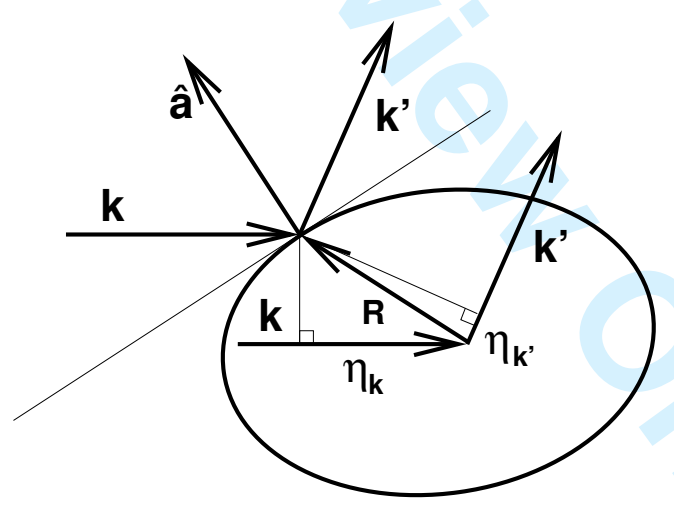

Figure 3: Calculating the phase shift. The path length of a scattering trajectory hitting the hardshell is compared to the path length of the same trajectory virtually hitting the centre of mass of the molecule (the starting point of the vector $\boldsymbol{R}$ ). The incoming part of the trajectory hitting the hardshell is $\hat{\boldsymbol{k}} \cdot \boldsymbol{R}$ shorter than the incoming part of the trajectory through the origin. This corresponds to a phase shift of $\eta_{k}=-\boldsymbol{k} \cdot \boldsymbol{R}$. Similarly, the outgoing part of the trajectory gives a phase shift of $\eta_{k^{\prime}}=\boldsymbol{k}^{\prime} \cdot \boldsymbol{R}$. The total phase shift is then $\eta=\eta_{k}+\eta_{k^{\prime}}$. See also equation (16). 
Figure 4: The hardshell PES for Ne-CO. The thick line is the hardshell, the dot in the middle is the centre of mass of the molecule, and the concentric, dashed circles connect points that are at the same distance from the centre of mass. The innermost circle touches the hardshell at the point of nearest contact and the following circles are all one de Broglie wavelength apart $\left(\lambda_{D}=0.63 a_{0}\right)$. This gives a good idea of the anisotropy of the hardshell. 


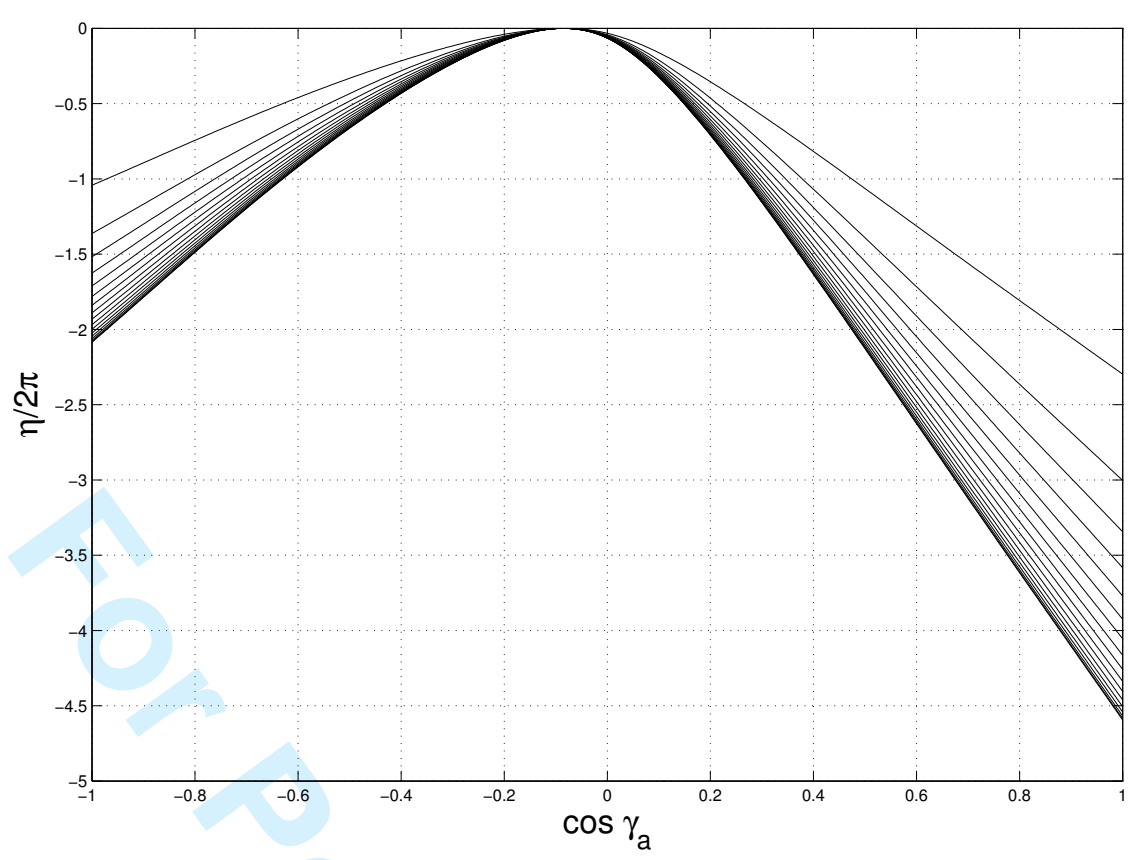

Figure 5: The phase shift curves for Ne-CO at $\beta=\pi$ (strictly backward scattering). They give the reduced phase, $\eta / 2 \pi$, as function of $\cos \gamma_{a}$. The curve nearest to the bottom is for the elastic case, $\Delta j=0$. These phases are different fromm those given by equation (16): instead of assigning the virtual trajectory through the centre of mass a phase shift of zero, the trajectory hitting the point of nearest contact is assigned a phase shift of zero. The O-end of the molecule is on the left side of the figure and the $\mathrm{C}$-end is on the right side, just as in figure 4 

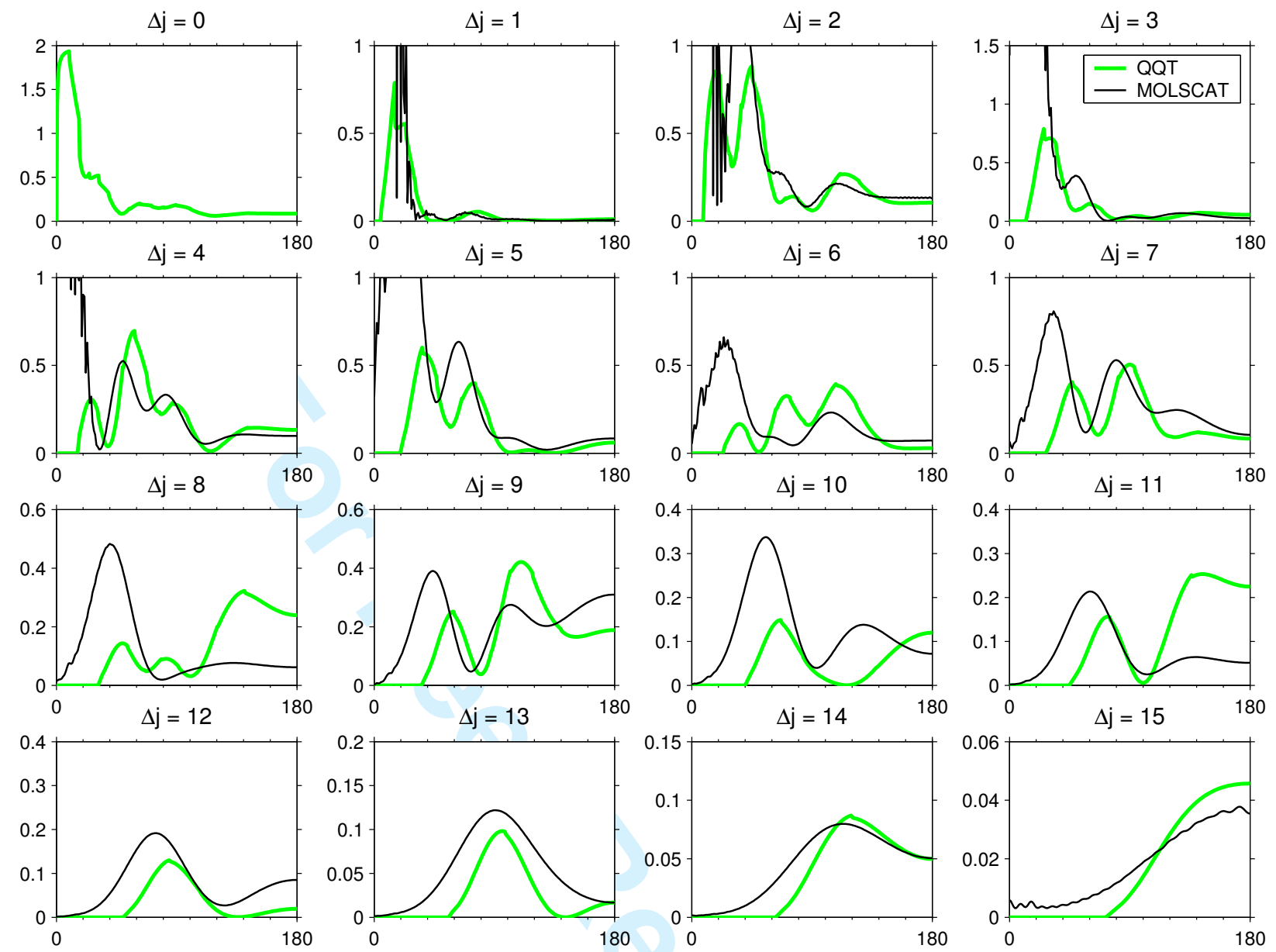

Figure 6: Ne-CO: a comparison between the DCS's from the QQT calculations using the $511 \mathrm{~cm}^{-1}$ equipotential line as a hardshell and the DCS's from MOLSCAT calculations using the full PES. The DCS's are given in $\left[\AA^{2}\right]$ as function of the scattering angle $\theta$ in $\left[^{\circ}\right]$. The value of $\Delta j$ is plotted above each subplot. 

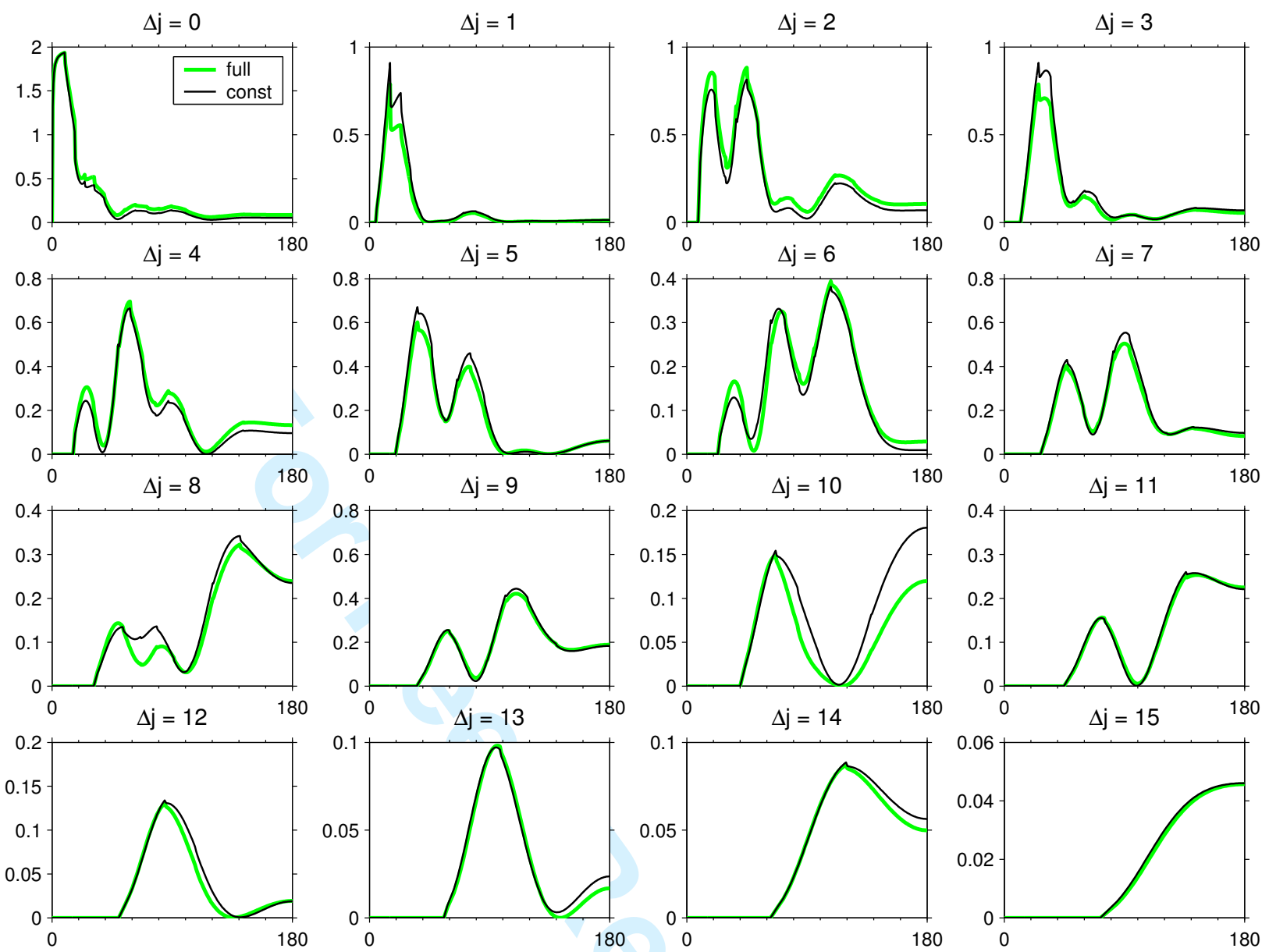

Figure 7: Ne-CO: a comparison between the DCS's from QQT calculations using the expression for the full curvature and the DCS's from QQT calculations using the constant curvature approximation. The DCS's are given in $\left[\AA^{2}\right]$ as function of [ $\left.{ }^{\circ}\right]$. 
Figure 8: The hardshell PES for He-NO. The thick line is the hardshell, the dot in the middle is the centre of mass of the molecule, and the concentric, dashed circles connect points that are at the same distance from the centre of mass. The innermost circle touches the hardshell at the point of nearest contact and the following circles are all one de Broglie wavelength apart $\left(\lambda_{D}=1.15 a_{0}\right)$. This gives a good idea of the anisotropy of the hardshell. 


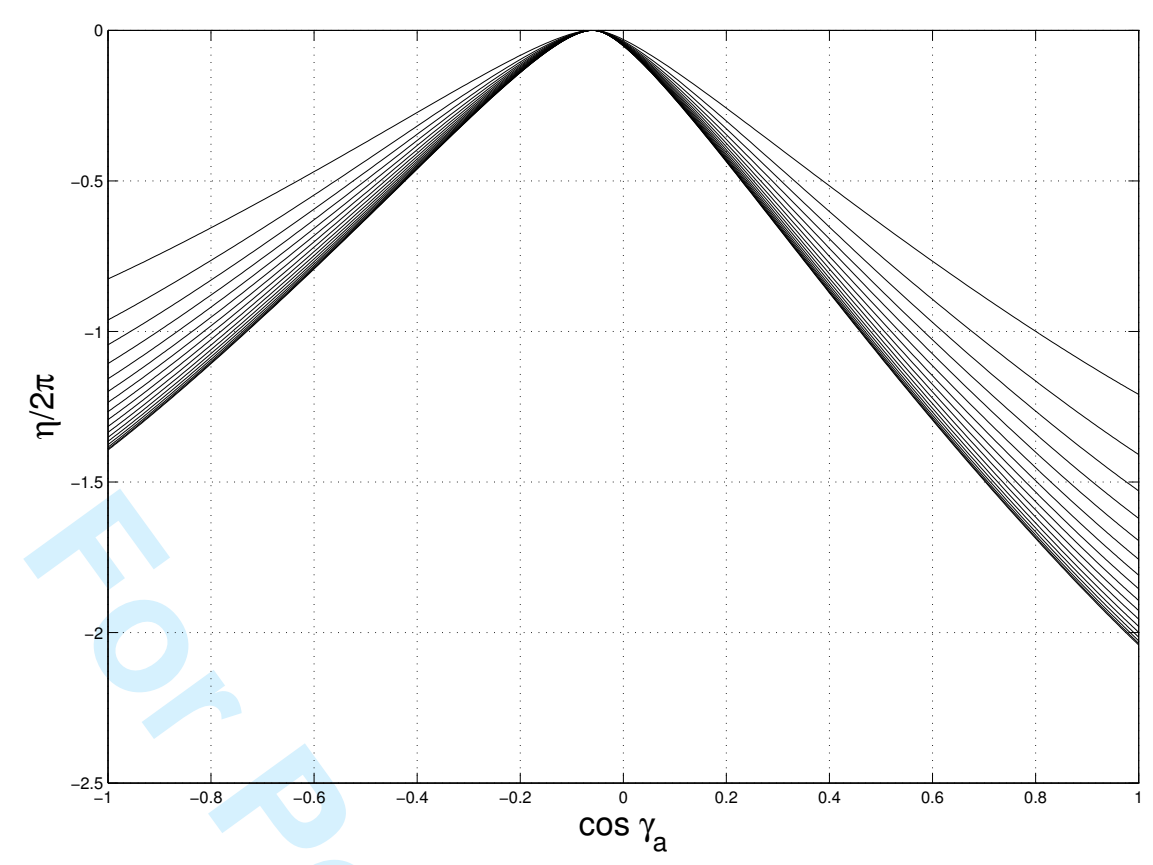

Figure 9: The phase shift curves for He-NO at $\beta=\pi$ (strictly backward scattering). They give the reduced phase, $\eta / 2 \pi$, as function of $\cos \gamma_{a}$. The curve nearest to the bottom is for the elastic case, $\Delta j=0$. These phases are different from those given by equation (16): instead of assigning the virtual trajectory through the centre of mass a phase shift of zero, the trajectory hitting the point of nearest contact is assigned a phase shift of zero. The O-end of the molecule is on the left side of the figure and the $\mathrm{N}$-end is on the right side, just as in figure 8 

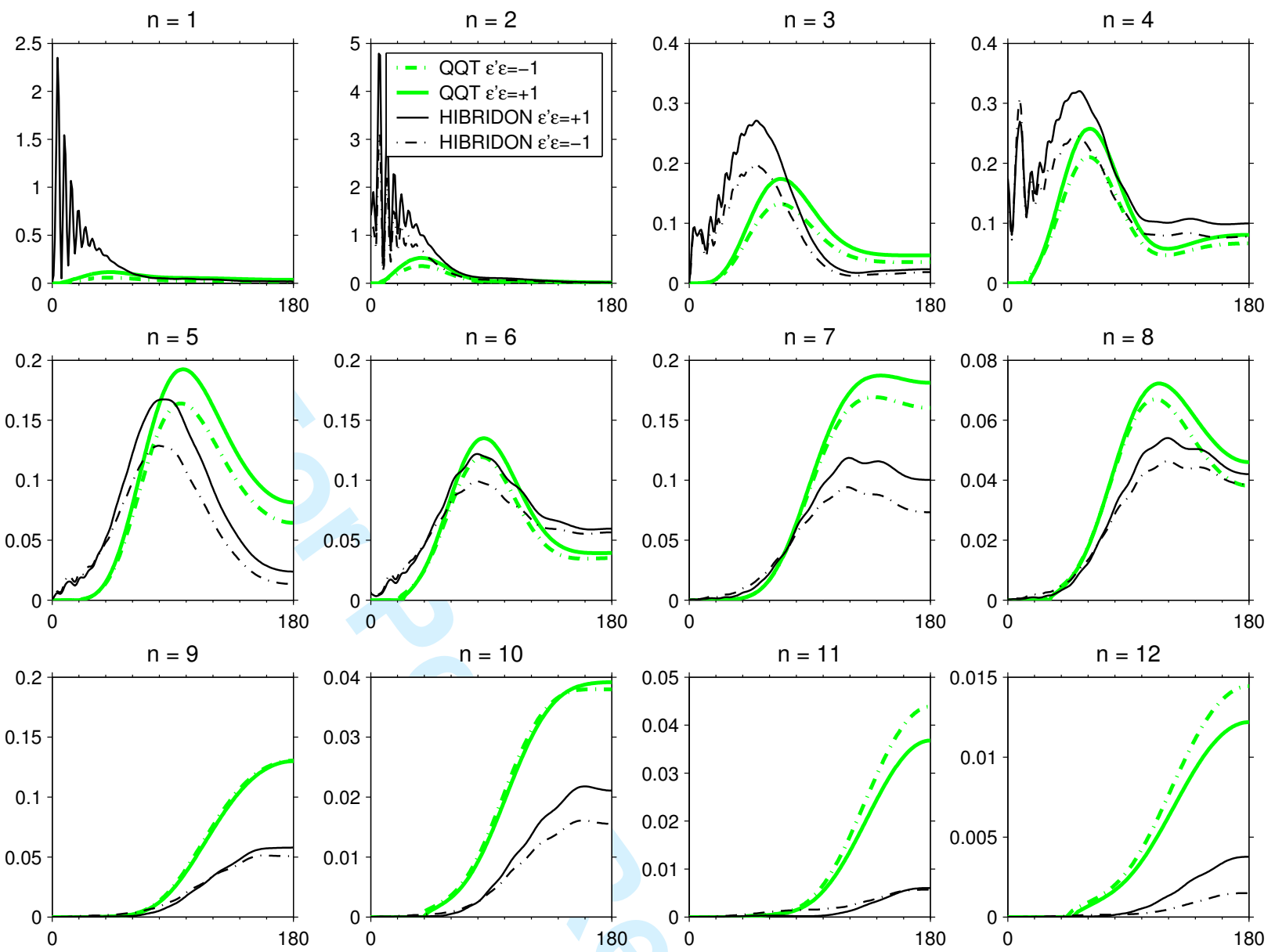

Figure 10: He-NO: a comparison between the DCS's from the QQT calculations using the 514 $\mathrm{cm}^{-1}$ equipotential line as a hardshell and the DCS's from HIBRIDON calculations using the full PES. The DCS's are given in $\left[\AA^{2}\right]$ as function of the scattering angle $\theta$ in $\left[^{\circ}\right]$. The value of the parity pair number $n=j^{\prime}-\frac{\epsilon^{\prime} \epsilon}{2}$ is plotted above each subplot. 

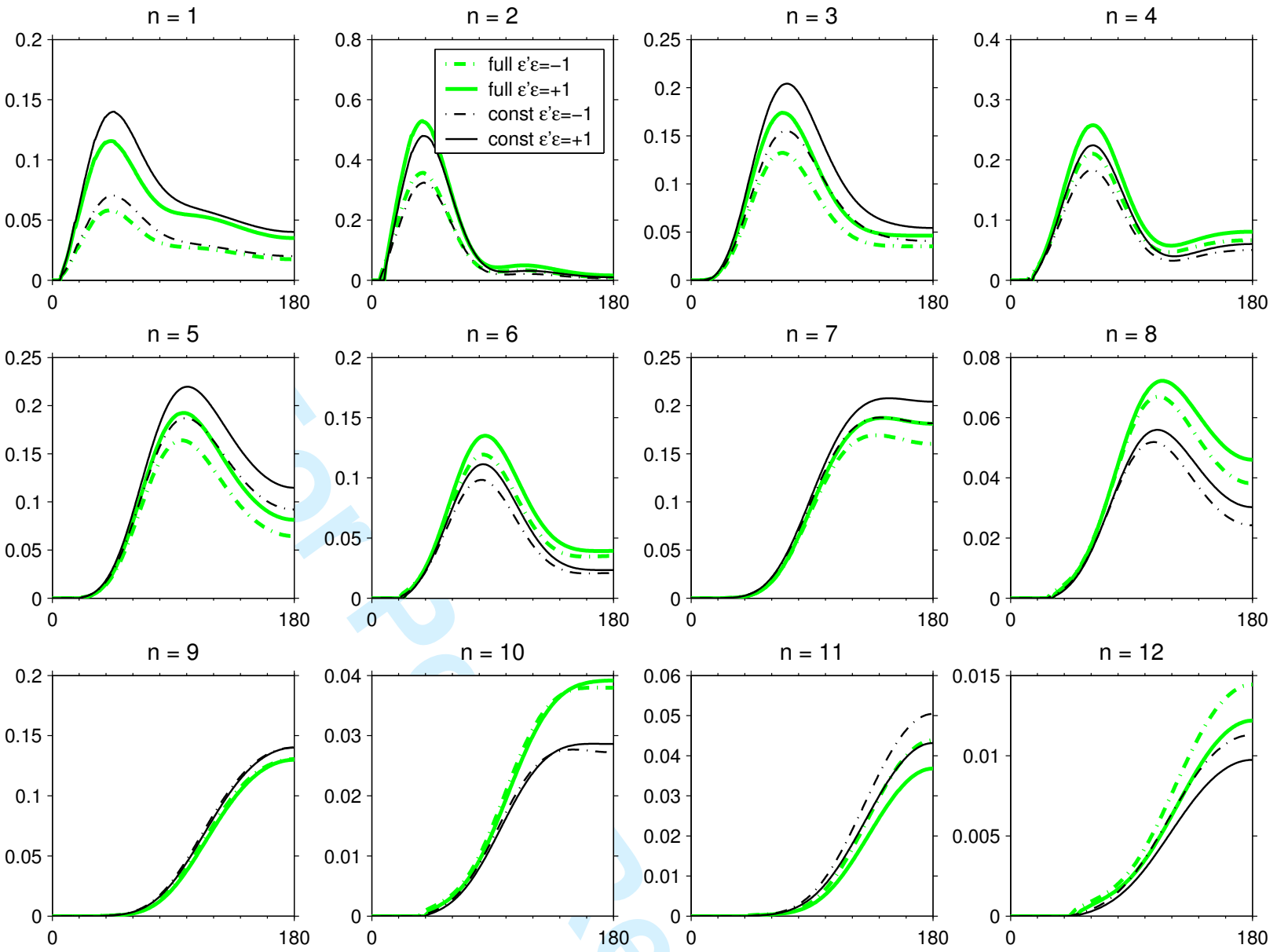

Figure 11: He-NO: a comparison between the DCS's from QQT calculations using the expression for the full curvature and the DCS's from QQT calculations using the constant curvature approximation. The DCS's are given in $\left[\AA^{2}\right]$ as function of $\left[^{\circ}\right]$. 


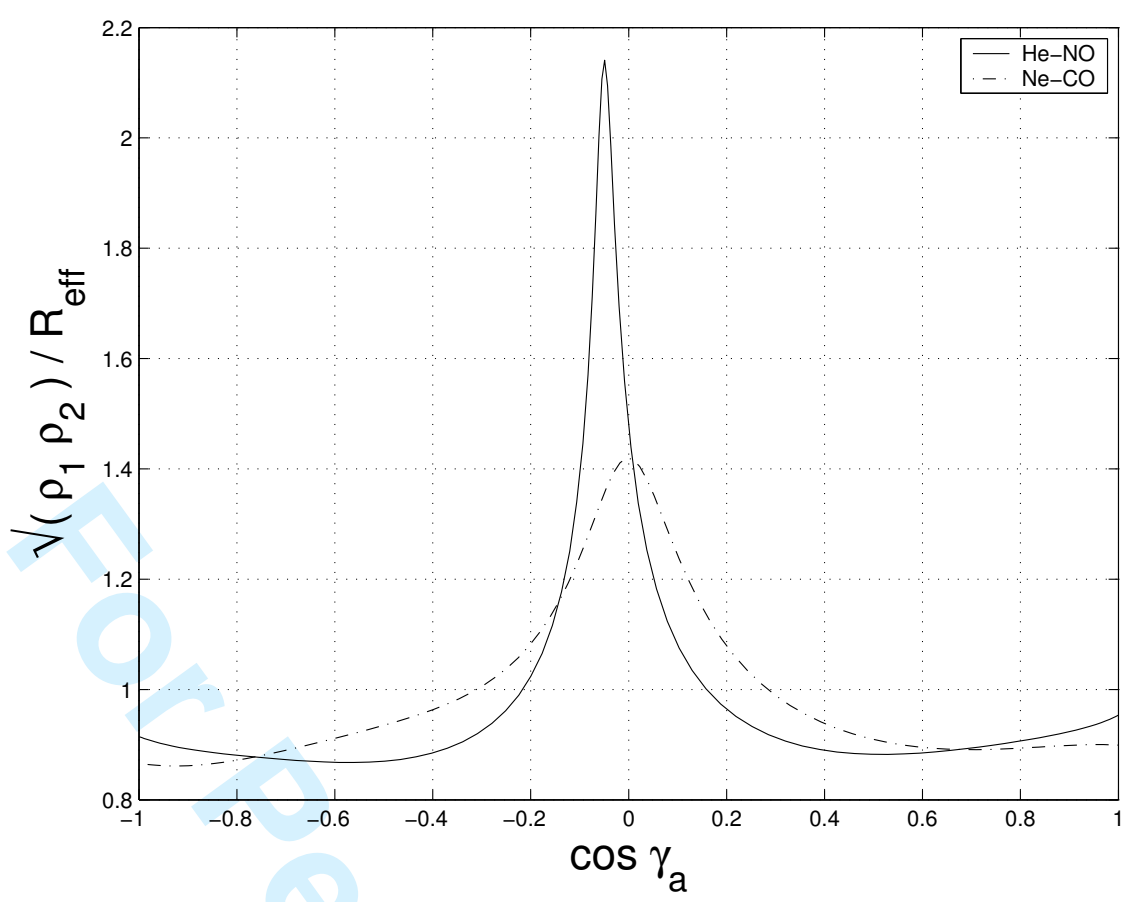

Figure 12: A plot of $\sqrt{\rho_{1}\left(\gamma_{a}\right) \rho_{2}\left(\gamma_{a}\right)} / R_{\text {eff }}$ as function of $\cos \gamma_{a}$ for both Ne-CO and He-NO. The O-end of the molecules is on the left side of the figure and the not-O-end is on the right side, just as in figures 4 and 8

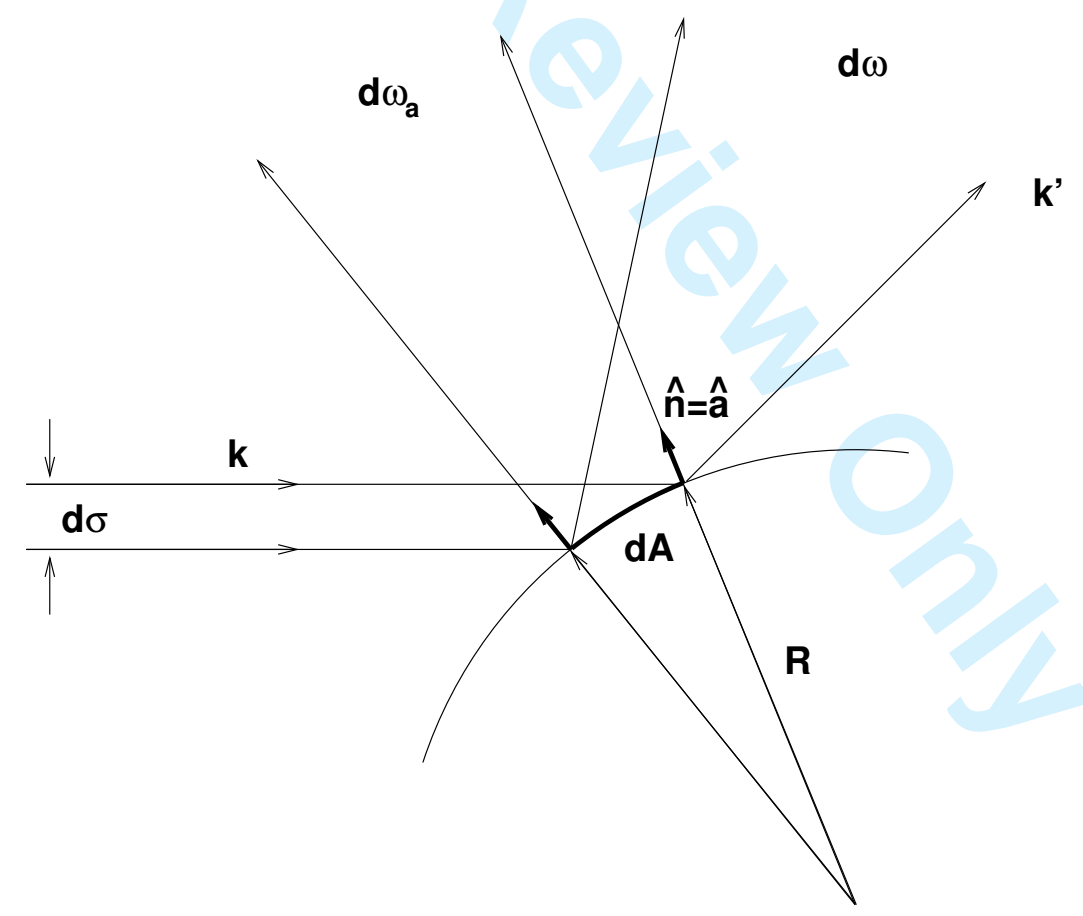

Figure 13: A schematic illustration of the geometrical differential cross-section. It shows why the DCS depends on the local curvature of the hardshell at the point of contact and it illustrates the relation between $d \sigma / d \omega$ and $d \sigma / d \omega_{a}$. 


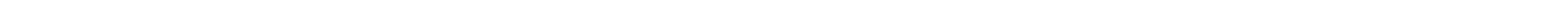




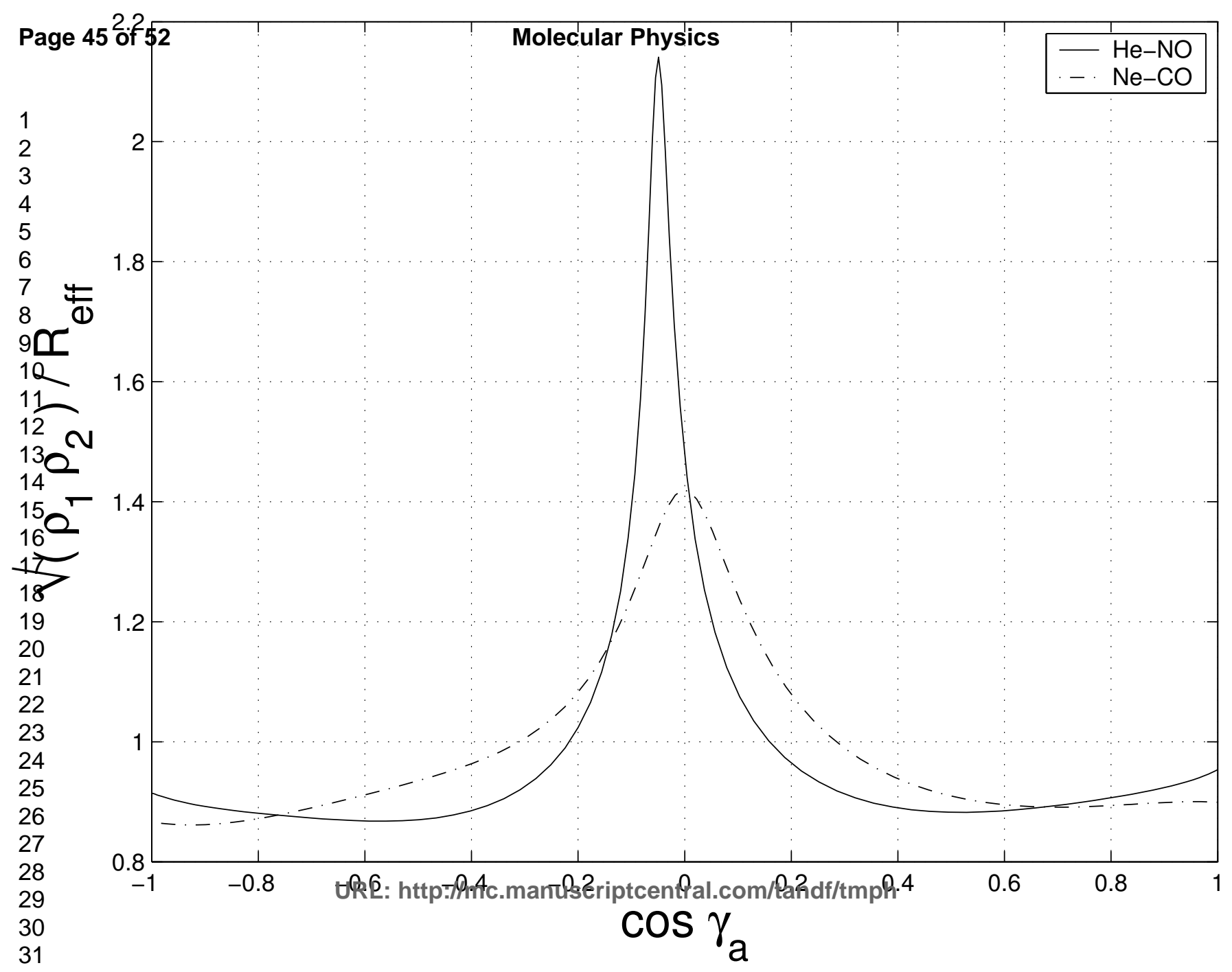


Page 47 of $52 n=1$
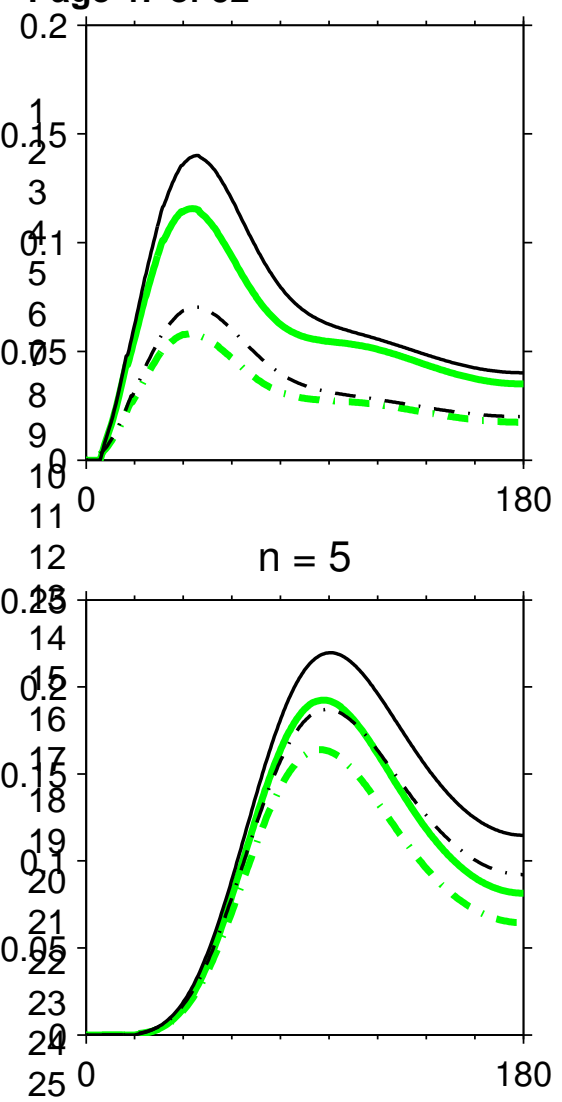

25

2

8
2
2
0.3
3
3
0
3
0.8
3
3
3
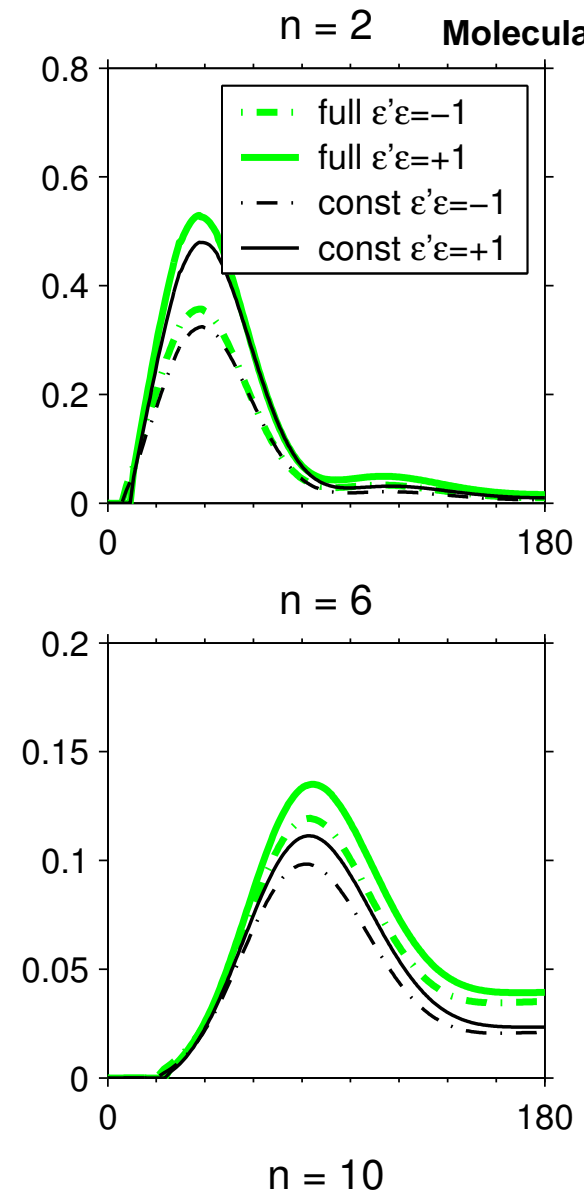
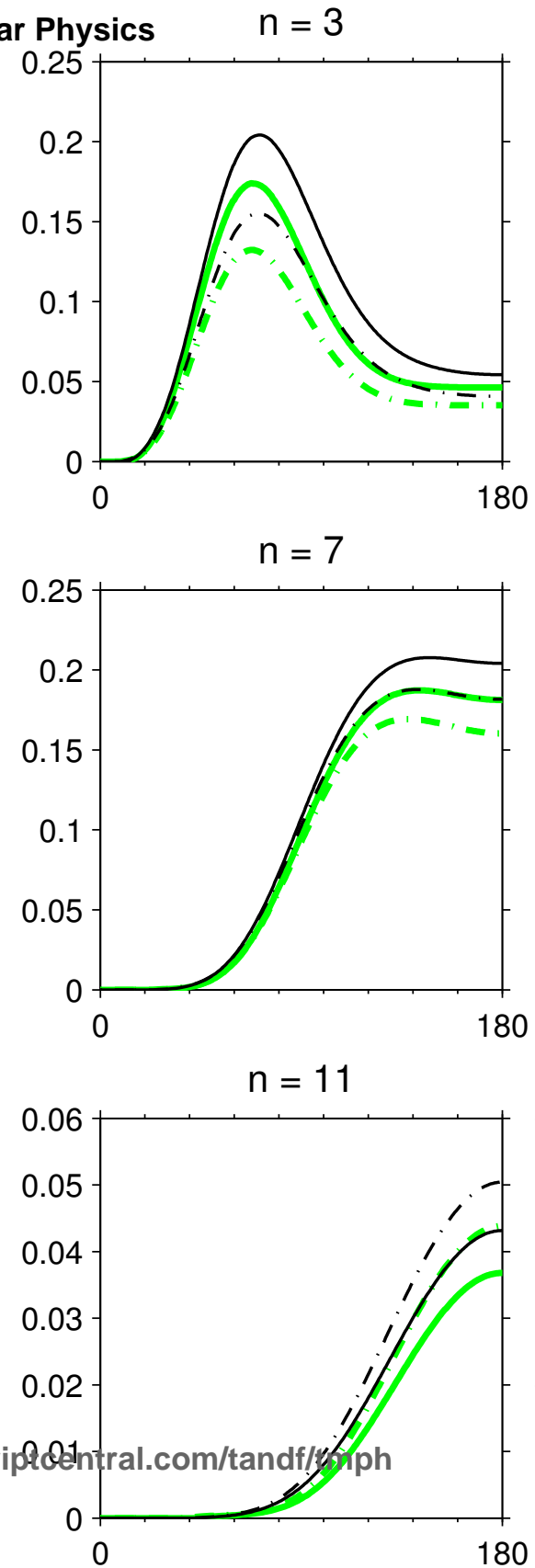
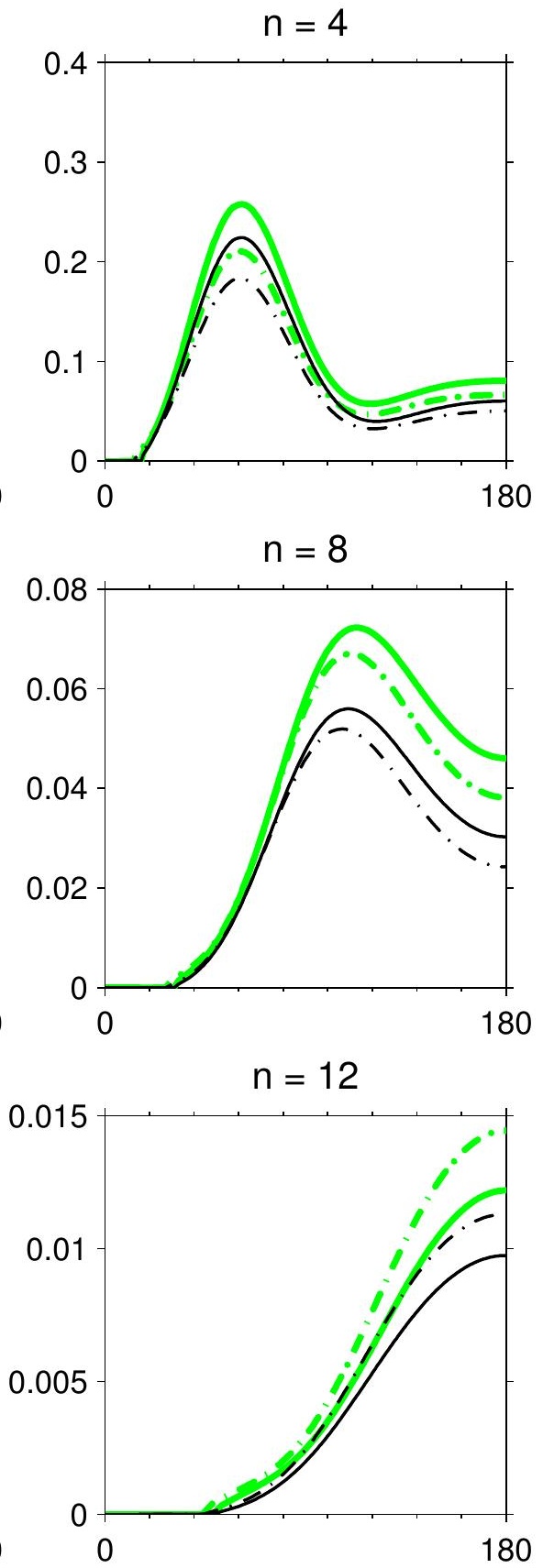


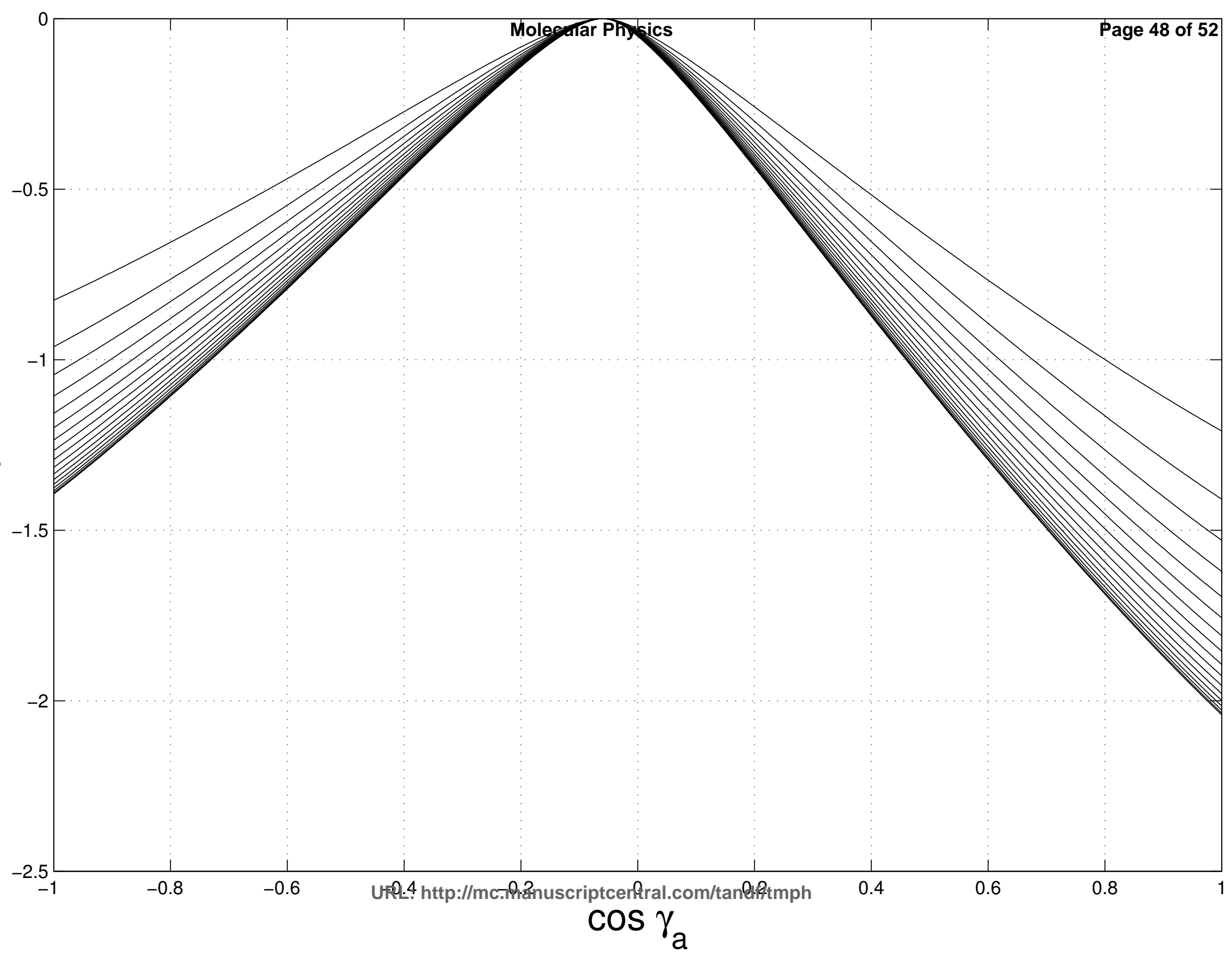




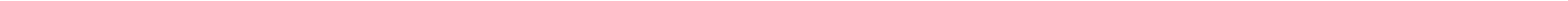


$d \omega$

$d \omega_{a}$

Molecular Physics

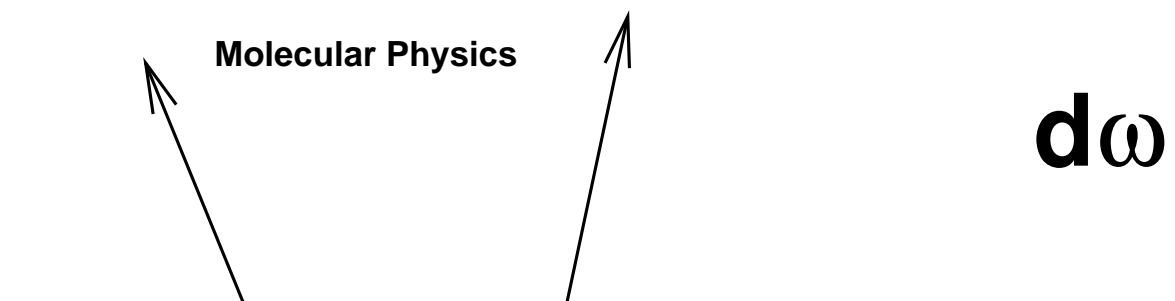

3

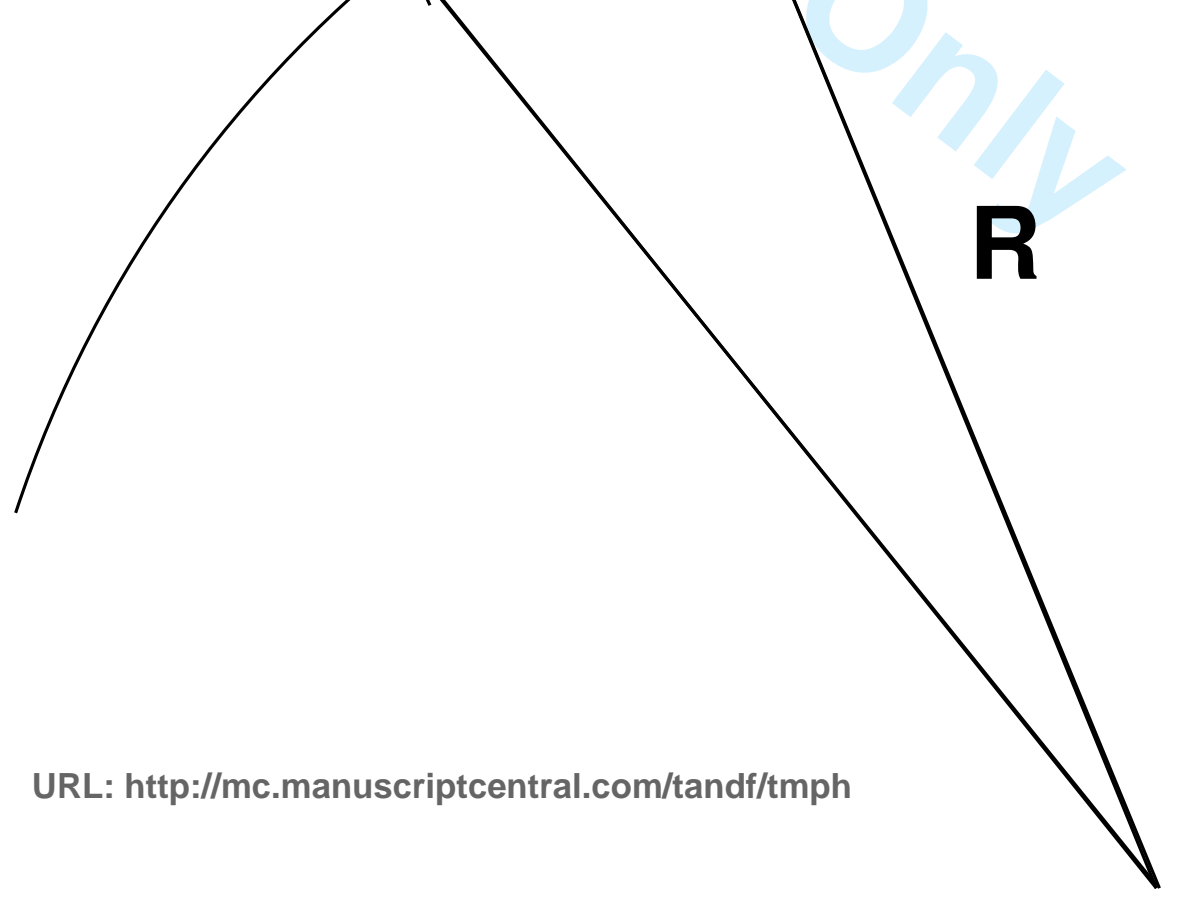




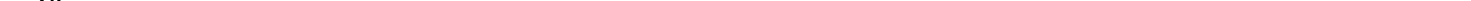




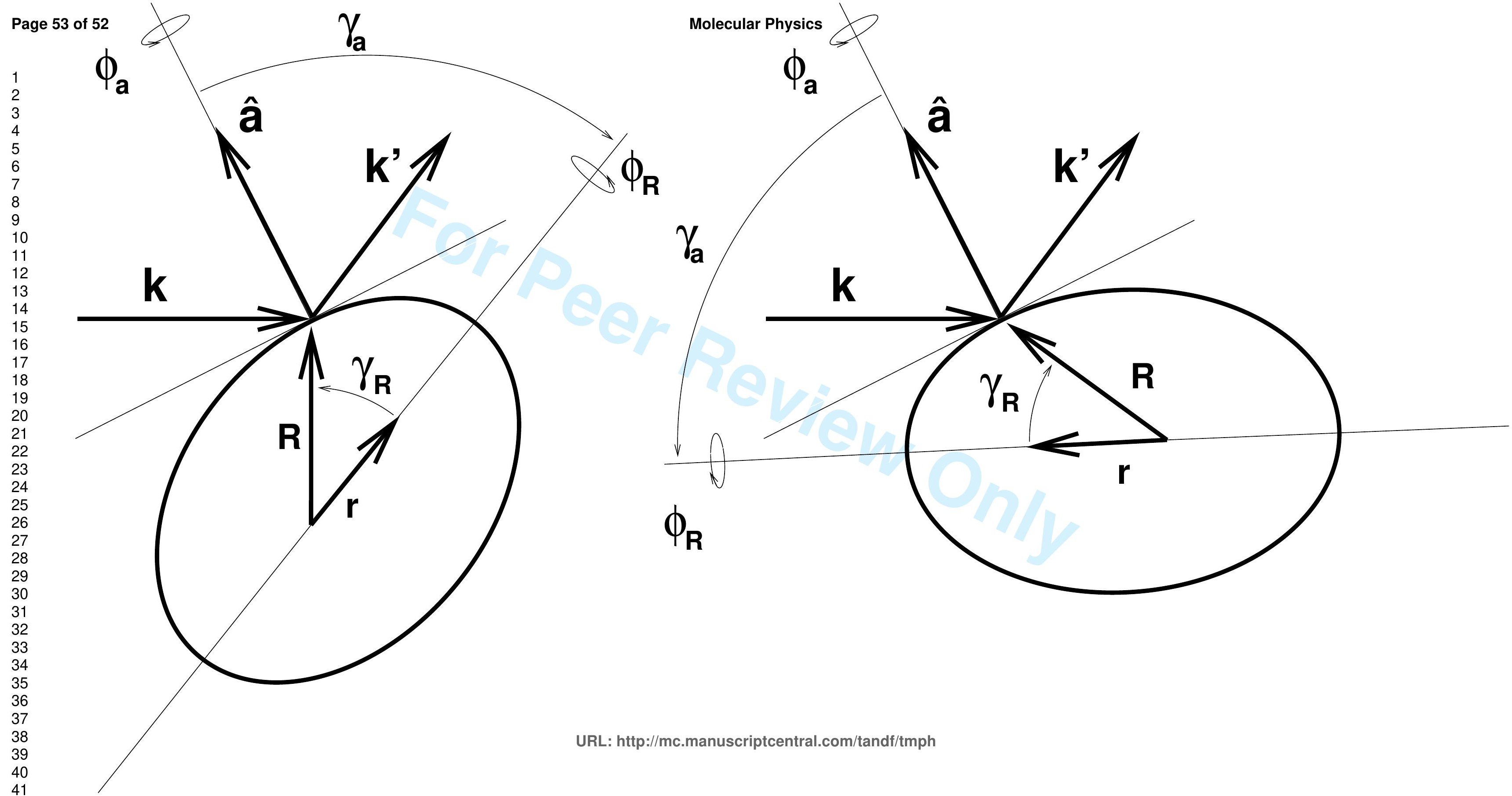

